NBSIR 81-2369

\title{
Residential Solar Data Center: Data Resources and Reports
}

U.S. DEPARTMENT OF COMMERCE

National Bureau of Standards

National Engineering Laboratory

Center for Bulding Technology

Washington, DC 20234

October 1981

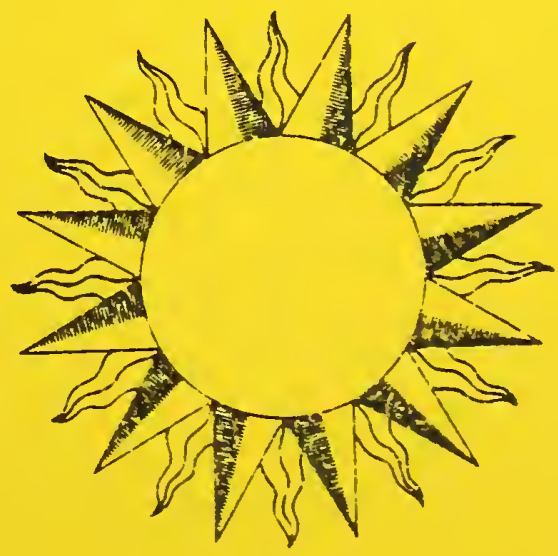

Prenared inr:

Department of Housing and Urban Development

ntfice or the Assistant Secretary for Policy Development and Research

- QC munnision of Energy. Building Technology and Standards

100 shington, DC $2041 \mathrm{C}$ 

‥

\section{RESIDENTIAL SOLAR DATA CENTER: \\ DATA RESOURCES AND REPORTS}

Patricia M. Christopher

Audrey 0 . Houser

U.S. DEPARTMENT OF COMMERCE

National Bureau of Standards

National Engineering Laboratory

Center for Building Technology

Washington, DC 20234

October 1981

Prepared for:

Department of Housing and Urban Development

Office of the Assistant Secretary for Policy Development and Research

Division of Energy, Building Technology and Standards

Washington, DC 20410

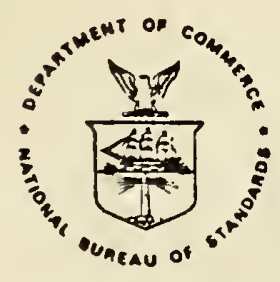

U.S. DEPARTMENT OF COMMERCE. Malcolm Baldrige, Secretary NATIONAL BUREAU OF STANDARDS, Ernest Ambler, Director 


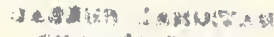

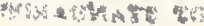

a तो

… 


\section{FOREWORD}

From January to October 1978 the Residential Solar Data Center (SDC) of the National Bureau of Standards (NBS) issued a bimonthly publication known as "Status Reports".[1] These reports contained sets of tables and charts designed to inform selected participants (primarily the Department of Housing and Urban Development and its contractors) in the Residential Solar Heating and Cooling Demonstration Program about the volume of data stored in the solar data base maintained by the SDC, and about the types of computer printouts that were available. The availability of computer printouts to a larger, more varied group of potential users was announced at the Department of Energy's Solar Heating and Cooling Systems Operational Results Conference held in Colorado Springs, Colorado, November 28 - December 1, 1978.

In June 1979 the document, "Residential Solar Data Center Data Resources and Reports,"[2] was published in an effort to enhance comprehension of the computer printouts of Residential Solar Demonstration Program data by this more general audience. Included was a summary of the history and background of the SDC and the demonstration program, an explanation of grant cycles and data collection procedures, and a full description of the files which comprise the solar data base.

The present document is an update to the June 1979 issue. It contains much of the same information, updated to reflect the current status of the SDC. This is the final update. As of the date of publication, the Residential Solar Data Center no longer exists. The data described in this report have been archived with their sponsor at HUD.*

\footnotetext{
*HUD User

P.0. Box 280

Germantown, MD 20767

Telephone: (301) 251-5154 
RESIDENTIAL SOLAR DATA CENTER:

DATA RESOURCES AND REPORTS

CONTENTS

Page

Foreword . . . . . . . . . . . . . . . iii

List of Tables................... v v

1. Background . . . . . . . . . . . . . . . . 1

Introduction to the Residential Solar Heating and Cooling Demonstration Program.

2. The Residential Solar Data Center . . . . . . . . 4 Introduction to the Solar Data Center; its development, its purpose, and its operation.

3. Summary of Computer Reports Available . . . . . . .

Summary of computer reports produced by the Solar Data

Center and the order in which report entries

are printed.

4. Sample Pages of Reports . . . . . . . . . . .

Sample pages illustrating format and content of

representative reports.

5. Data File Elements . . . . . . . . . . . .

Detailed description of the files which comprise the solar data base.

6. Interactive Access to Solar Data . . . . . . . .

Description of the query package available to facilitate online access to interactive files contained in the solar data base.

7. Amount of Data on the Computer . . . . . . . .

Figures indicating by cycle the extent to which

computerization of data has been accomplished.

8. Acknowledgments . . . . . . . . . . . . . 58

9. References.................. 59 


\section{LIST OF TABLES}

Page

Table 1. Summary of Grant File Computer Reports . . . . 8

Table 2. Summary of Grantee File Computer Reports . . . 9

Table 3. Summary of Technical Description File Computer

Reports ................... 9

Table 4. Summary of Technical Concerns File Computer

Reports ............... 10

Table 5. Summary of Marketing Survey File Computer

Reports ................. . 11

Table 6. Summary of Ctility Consumption File Computer

Reports ................. . 11

Table 7. Summary of Grant File Data . . . . . . 52

Table 8. Summary of Grantee File Data . . . . . . 53

Table 9. Summary of Technical Description File Data - 54

Table 10. Summary of Technical Concerns File Data . • . 55

Table 11. Summary of 'Sarketing Survey File Data . . . 56

Table 12. Summary of Utility File Data . . . . . . 57 


\section{Residential Solar Data Center: \\ Data Resources and Reports \\ Patricia M. Christopher Audrey 0. Houser \\ National Bureau of Standards \\ Washington, D.C. 20234}

The Residential Solar Data Center (SDC) was responsible for the establishment and operation of a computerized data base containing non-instrumented residential data collected from the DoE/HUD Solar Heating and Cooling Demonstration Program. This document includes a summary of the history and background of the SDC and its role in the demonstration program, a list of the final computer reports which are available, sample pages of representative reports, and a description of the data files which comprised the solar data base.

Key words: automatic data processing; data base; residential buildings; solar data base; solar heating and cooling; solar energy systems. 


\section{BACKGROUND}

In 1974, Congress passed the Solar Heating and Cooling Demonstration Act to establish a program of research, development, and demonstration directed towards reducing the nation's dependence upon non-renewable resources through stimulating the development and use of solar energy systems. The Department of Energy (DoE) is responsible for the management of the total Federal Solar Energy Research, Development, and Demonstration Program. DoE was assisted in the demonstration portion of the program (which terminated in 1981) by the Department of Housing and Urban Development (HUD), the National Bureau of Standards (NBS), and other Federal agencies and private contractors.

The demonstration program was divided into two parts: a Residential Program for which HUD had prime responsibility; and a Commerical Program, directed by DoE. In both programs, funds were allocated for new and retrofit building projects in a variety of climatic and geographic regions. These projects were designed to demonstrate the economic viability of the use of solar energy systems for heating and cooling.

A principal objective of the demonstration program was to provide data on the technical aspects of solar energy systems and on their acceptance by the building industry, regulatory agencies, and the consumer. Data were collected in two ways: manually (non-instrumented data) and electronically (instrumented data).

Non-instrumented data, technical and non-technical, were collected on questionnaires or take-off forms for entry into the computer.

Included were data describing the demonstration projects and their solar energy systems as well as data concerning the progress of the grant from construction through marketing, market acceptance, etc.

Instrumented technical data were derived principally from sensors installed when construction activities were completed at selected project sites. These data, when analyzed, defined the thermal performance of the solar energy systems and the climatic conditions affecting that performance.

DoE contractors were responsible for the collection of both instrumented and non-instrumented data in the commercial program and for the collection of instrumented data only in the residential program. The responsibility for collection of non-instrumented data in the residential program resided with HUD. Figure 1 illustrates the assignment of data collection, evaluation, and dissemination responsibilities in the Solar Demonstration Program. The Residential Solar Data Center (as shown in figure 1) was the entity responsible for storage, retrieval, and dissemination of non-instrumented solar data in the residential program. 


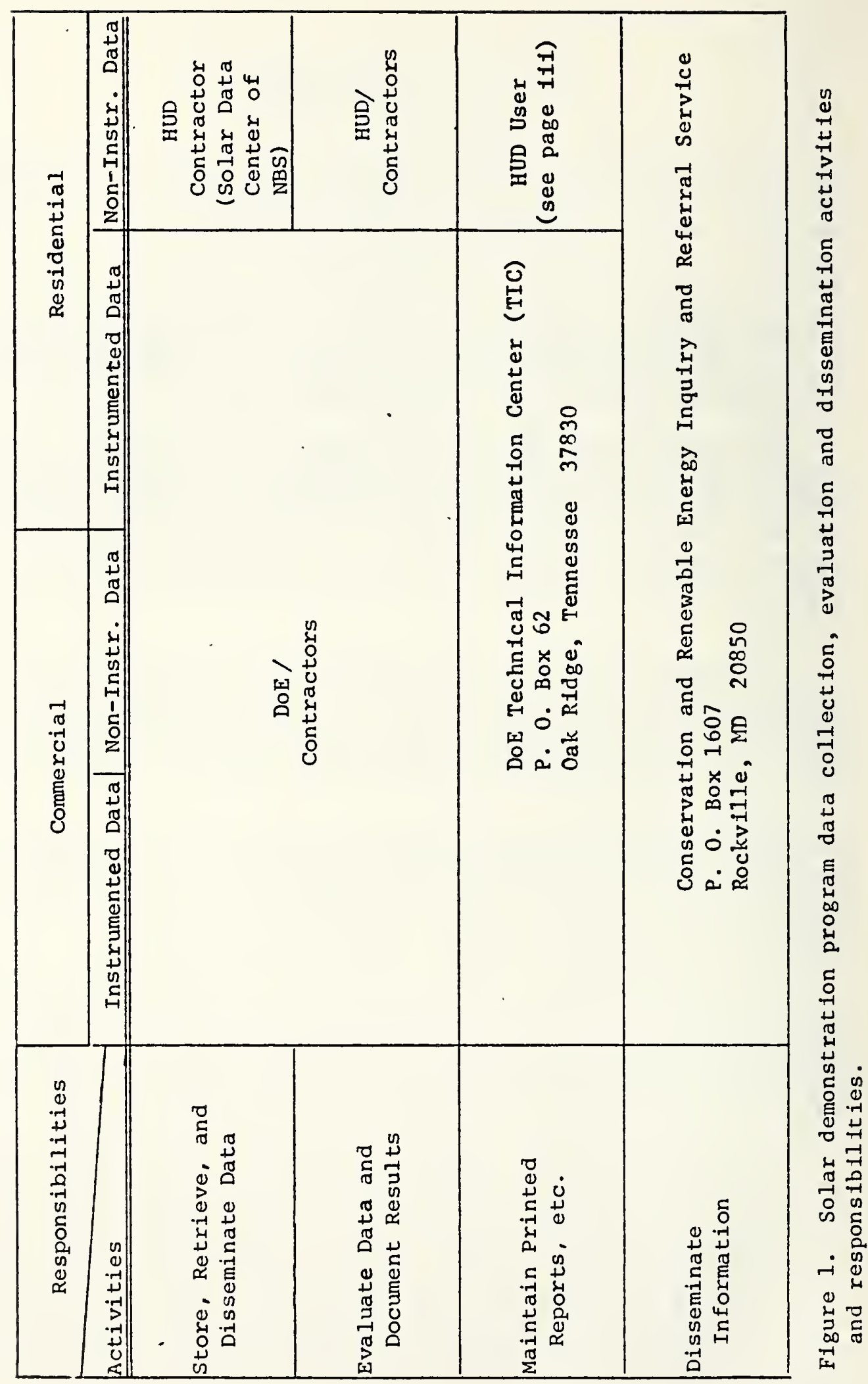


In implementing the Residential Demonstration Program, HUD had established four main objectives. They were:

1. Residential demonstrations of solar equipment;

2. Development of performance criteria and certification standards for solar equipment;

3. Encouragement of the acceptance and use of solar technology by the housing industry and the general public; and

4. Dissemination of demonstration and market development data.

In order to accomplish these objectives, HUD funded demonstration projects (by awarding grants) in six cycles initiated approximately every nine to twelve montins.* Data collected from funded projects in each cycle enabled HUD and its contractors to apply increased awareness of solar technology, marketplace dynamics, and data collection techniques towards enhancing the effectiveness of projects in succeeding cycles.

In addition, HUD, in cooperation with DoE, had established a national clearinghouse and reference center for the effective dissemination of information regarding solar energy systems - technical and non-technical, domestic and foreign, residential and commercial. The center functioned as a major reference resource for all elements of the solar community, as well as for the general public. The latest demonstration information was made available by the center through publications, conferences, and exhibits and through its toll-free telephone and national mailing response mechanism.

*For a count of grants per cycle, see section 7 . 


\section{THE RESIDENTIAL SOLAR DATA CENTER}

In the Fall of 1976, the design for a solar data center was initiated by the Institute for Computer Sciences and Technology (ICST) at the National Bureau of Standards (NBS). A series of publications [3] developed the framework for what was to become the Residential Solar Data Center (SDC).

In March 1977 the SDC became operational at NBS and operated until October 1981 when the program ended. The SDC was responsible for the establishment and operation of a data base containing non-instrumented solar data collected by participants in the Residential Solar Demonstration Program which was managed by the Department of Housing and Urban Development (HUD).

The principal data collection contractor for HUD in the demonstration program was the Boeing Aerospace Corporation (BAC) which had subcontracted with the American Institute of Architects/Research Corporation (AIA/RC); Dubin, Bloome Associates (DBA); and the Real Estate Research Corporation (RERC). These contractors collected and forwarded data to the SDC which maintained a solar data base consisting of the following files:

1. Grant File: This file contained basic project and system information for each application funded by HUD. These data were derived from grant applications submitted to HUD and updated with information from periodic field reports. More detailed information about this file is available in [4].

2. Grantee Report File: Data in this file were based upon reports submitted by each grantee to BAC describing the progress of the grant from design and award of construction financing through actual construction, sale, and permanent financing. The grantee's perception of the ease or difficulty in obtaining construction or permanent financing and building and zoning approval, as well as construction, equipment, or installation problems were included.

3. Technical Description File: This file contained basic system design and predicted performance data collected by DBA from a large number of selected non-instrumented systems and for all instrumented systems. A more detailed set of data was collected by AIA/RC for those systems which were instrumented. The data sample corresponded to the marketing survey and the utility consumption samples.

4. Technical Concerns File: Contained in this file were data on problems found during the design, construction or operational 
Dhase which were recorded in field activity reports submitted by $D B A$ and BAC field representatives. It also contained data on problems found after construction, as recorjed by the grantee.

5. Marketing Survey File: This file contained extensive survey questionnaire results collected by RERC from silected builders, lenders, homebuyers, code officials, utility companies, and other market participants. The data sample included representatives of those who chose to build, lend, or buy a funded solar house and "comparatives" who did not become involved. Data were also collected after the sale to gause builder and consumer reactions over a period of time. The data sample selected for these surveys (about 25 percent of all grants) is the same sample for which technical description data and utility consumption data were collected.

6. Utility Consumption Eile: This file contained information on auxiliary or "backup" fuel consumed for selected projects. The projects selected were those for which marketing survey results were collected. The data were collected from utility companies (with purchaser agreement). "Comparative" data were also collected.*

The following is a brief description of the services which were provided by the SDC:

\section{Receipt and Maintenance of Data}

The SDC provided a central location for the receipt, storage, processing and reduction of non-instrumented, residential solar data collected from the Solar Demonstration Program. Data were collected and transcribed onto computer forms by HUD and its contractors. These forms were sent to the SDC and from there to NBS contractors who keyed the information into machine-readable formats. The incoming data were then edited, catalogued, reformatted, translated, and validated. These activities provided the necessary control and prepared the data for use in the production of appropriate reports.

\section{Production of Printed Reports}

A major function of the SDC was the production of reports ranging from complete listings of all data in a file to more dctailed "custom" computer reports. Custom reports were produced to meet specific user requirements and may have printed only selected data from a file and may have re-sorted the selected data into a new sequence. New report

\footnotetext{
* A more complete description of data files and specific data elements is contalned in section 5 .
} 
requirements were defined by HUD or its contractors in the Residential Demonstration Program. These reports were generated whenever a data file was updated.*

\section{Provision of Online Access to the Data}

Some data files could be accessed by authorized users (as determined by HUD) via a computer terminal. Access was made possible through the use of a query package called MIRADS (Marshall Information Retrieval and Display System).

\section{Ad Hoc Functions}

In addition to the operation and maintenance of the data base, the SDC also provided the following user services (see [4], [5]):

1. technical expertise to answer user questions and to provide assistance;

2. development of computer programs in response to users' special needs;

3. user training in online access to the data base and in procedures for transcription and validation of data;

4. documentation of available data, reports, and online access techniques;

5. interface with data collectors and users;

6. development of standards for terminology, programs, and documentation.

7. archiving of files, computer listings, documentation, and programs.

*See section 3 for a list of the final computer reports available from HUD. 


\section{SUMMARY OF COMPUTER REPORTS AVAILABLE*}

The tables in this section show final computer output reports which are available from HUD. (See address on page iii.) Tables $1-6$ summarize content, and indicate report sequence (i.e., sorted by grant number, sorted by manufacturer, etc.). Sample pages from all these reports are contained in section 4 .

The following is an explanation of terms used in the headings of tables 1 - 6:

Description: A brief description of the data elements included in each report. (See section 5 for additional information.)

Sequence of Data: The order in which line items are sorted.

Report Number: Number by which the report is referenced when requesting a copy from HUD. This same number appears at the top of each page of the report.

*Summary statistics of Grant, Grantee, Technical Description, and Utility Consumption file data are currently being compiled and will be avallable from the Department of Housing and Urban Development (HUD). 
Table 1. Summary of Grant File Computer Reports

Description

Sequence

Number

of Data

of Report

Most of these computer reports are available in [4].

Complete listing of all data collected for each grant awarded. (492 pages)

Grant

SG-C 1

Number

Analysis of units and costs for grant awarded showing average unit cost. ( 1 page)

System

SG-C2

Type

Listing of grants awarded with grantee name, project city and state, housing type, con-

Grant

SG-C 3 struction type, dwelling count, solar system, number of buildings, system type, system kind, collector sq. ft., designer cost to government, builder cost to government, solar fraction, and solar manufacturer. (25 pages)

Same as SG-C3 except auxiliary fuel type and storage medium are shown instead of solar fraction. (25 pages)

Same as SG-C3 except collector sq. ft. and cost per MBtu is shown instead of designer and builder costs to government. (25 pages)

Same as SG-C3 except cost to government is shown instead of designer and builder costs to government and solar fraction. (32 pages)

Same as SG-C3 except grantee city and state are shown instead of project city and state. (28 pages)

Number

Grant

$S G-C 3 F$

Number

Manu-

SG-C 4BC

facturer

Manu-

SG-C 4CG

facturer

Same contents as SG-C3. (28 pages)

Grantee

SG-C5AS

City and

State

Project

City and

State

Same as SG-C3 except HUD region is also shown. (26 pages)

HUD

$S G-C 7$

Region 
Table 2. Summary of Grantee File Computer Reporzs

$\begin{array}{lll} & \text { Seque }=c e & \text { Number } \\ \text { Description } & \text { of } D= \pm a & \text { of Report }\end{array}$

Listing of all Grantee Report data. (500 pages) Gran=əe BA-R1

Repo:=,

Card liumber,

Proj $==t$ ID

One page per project of all Grantee

Report 1,3 and 4 data, with field titles.

Proje=t ID $B-R 2$

(937 pages)

Listing of all project IDs for each grantee report in the data base. (19 pages)

Proj $=2 t$ ID $B-P 4$

Table 3. Sumary of Technical Description File Compute= Reports

Description

Sequince Number

of $\mathrm{D}=\mathrm{a}$ of Report

Listing of data on instrumented and

non-instrumented systems for which F-Chart

calculations were done. This listing includes

the F-Chart input and output data. (200 pages)

Proje=t ID, DA-RI

Systeב

Number

Listing of data on instrumented systems

including:

- the site and building with a solar system

- the collector subsystem

- the thermal storage subsystem

- the controls subsystem

- the circulation subsystem

- the auxiliary energy subsystem, and

- the predicted system performance.

$(10,000$ pages $)$ 
Table 4. Summary of Technical Concerns File Computer Reports

Listing of all technical concerns data for each system with problems. Codes are translated for hardware element, actions, and events. (223 pages)

Grant

Number,

Sys tem

Number,

Date

Simplified listing of $C B-D 3$, with hardware element and events data in "summarized" form. Additional data shown is cycle, number of units, new or retrofit, housing type, system type, system kind, and transfer medium. (67 pages)

Summary of hardware element with technical concerns. (22 pages)
Grant

CB-D 3A

Number, System Number

Grant CB-HAS

Number, System

Number 
Table 5. Summary of Marketing Survey File Computer Reports

$\begin{array}{lll} & \text { Sequence } & \text { Number } \\ \text { Description } & \text { of Data } & \text { of Report }\end{array}$

Question and answer dictionary, showing abbreviated forms for all marketing survey questions and all the possible answers, both coded and uncoded. (463 pages)

Question- RA-RI thru naire ID, $R Z-R I$

Question

number, Project ID

Iisting of all answers for the marketing survey questionnaire from single family builder thru follow-up comparative renter. (200 pages)

Questionnaire ID, Question number, Project ID

Table 6. Summary of Utility Consumption File Computer Reports

Description
Sequence of Data
Number of Report
Listing of all utility consumption data (usually monthly) and comparative data, including averages of fuel usage for each unit. (650 pages)

Listing of only the yearly average fuel usage data for each unit. (15 pages)

Listing of utility supplier data for those companies supplying fuel to units. Report includes name and address of supplier, as well as code by which data was stored on computer. ( 6 pages)
Project ID, BF-R I

Fuel type,

Billing

Start date

Project ID, BF-RI-AVG

Fue1 type

Utility

supplier

code
RA-2 thru

$\mathrm{RZ}-2$ 


\section{SAMPLE PAGES OF REPORTS}

This section contains copies of actual pages from each report. Since these examples may contain out-of-date or out-of-context data, they should be viewed as "samples" only. The title of the report, the date it was produced, the report identification number, and sequential page number are shown on the top of each page. An explanation of "not applicable" codes (as seen on pgs. 22 and 23) is given below. Other codes may be directly interpreted since meaningful abbreviations were used whenever possible. Codes are more fully explained with the complete report.

\section{"Not Applicable" Codes}

Missing data in these reports were usually indicated by one of four "not applicable" codes. The four codes and their translations are shown below. When space is available in the report, the code was translated and only the interpretation was printed.

\section{N/A Code}

$\mathrm{XX}$

$X A$

$\mathrm{XB}$

$\mathrm{XC}$

\section{Translation}

Information will be available later Information will not be collected Information not required See additional comments 


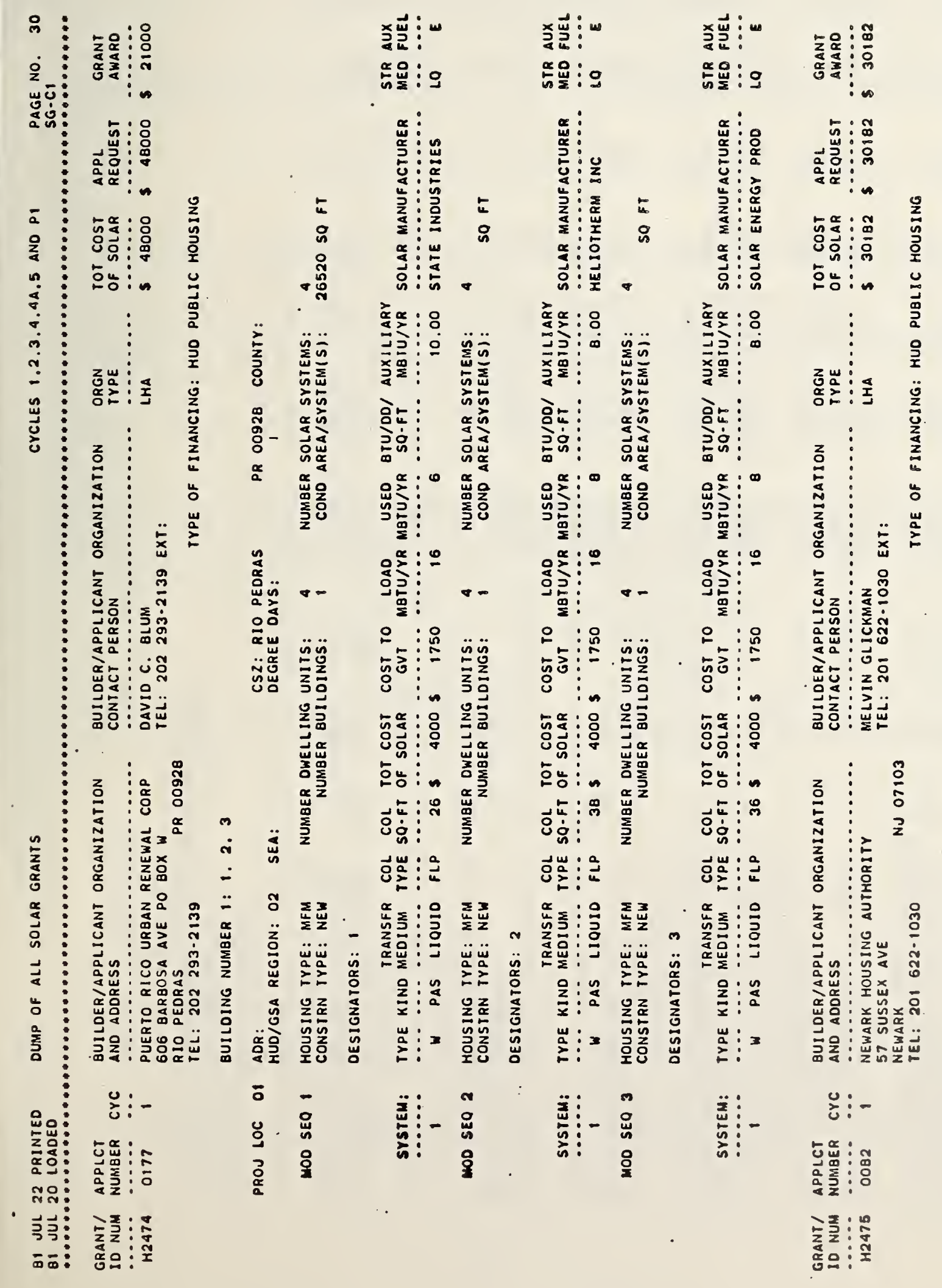


-

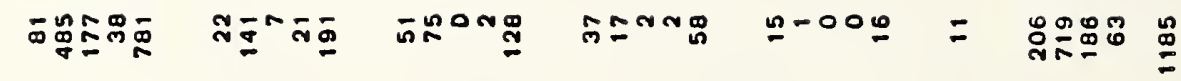

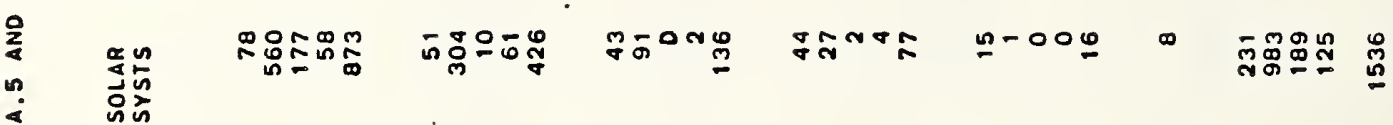

战定

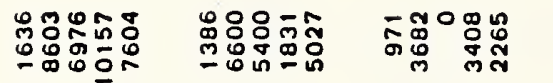

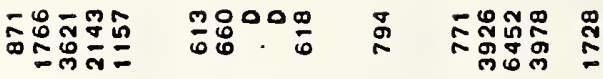

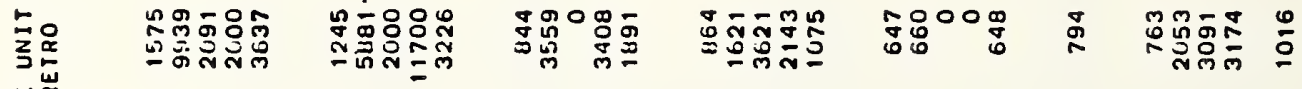

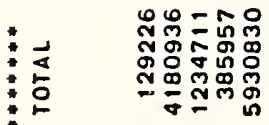

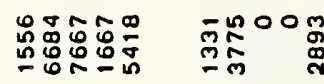

: ํํㅇํํ요

串 $000 \%$

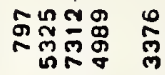

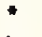

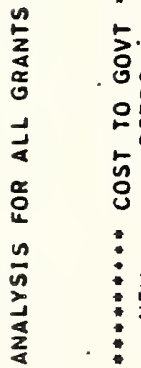

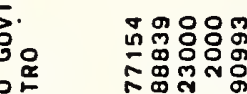

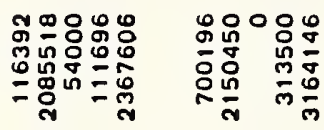

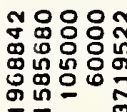

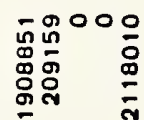

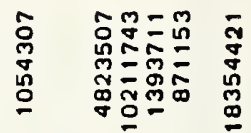

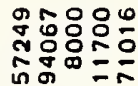

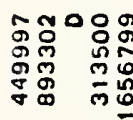

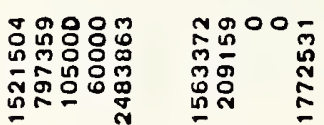

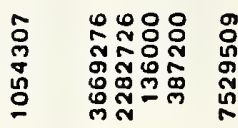

$\frac{3}{2}$

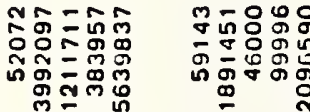

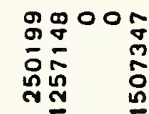

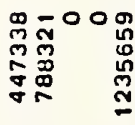

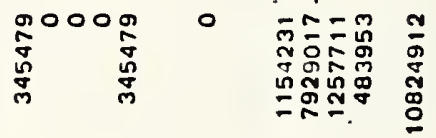

:

安

*

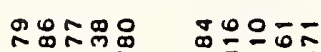

ลัฐัํำ

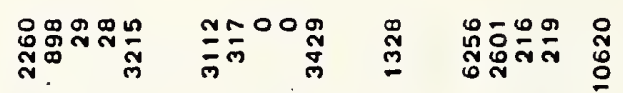

产

$\stackrel{n}{\frac{2}{3}}$

要

온연

สํำ

引亏

$\overline{\bar{\omega}} \bar{\sigma}$

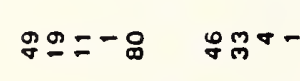

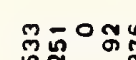

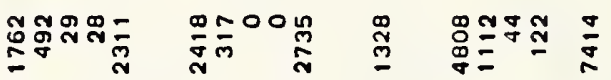

ㅇํํำำ

零

罵

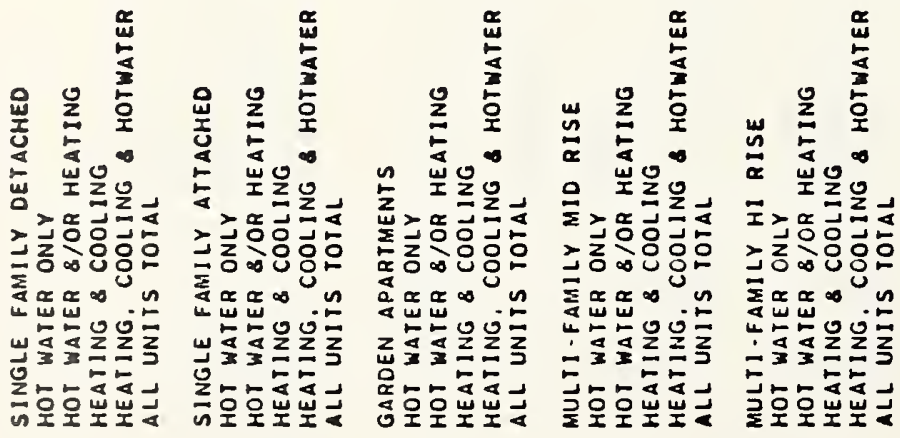

잉유

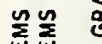

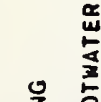

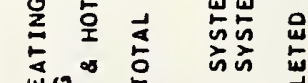

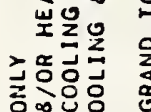

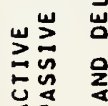

का \&

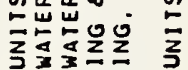

งᄂ的

แั

똡 


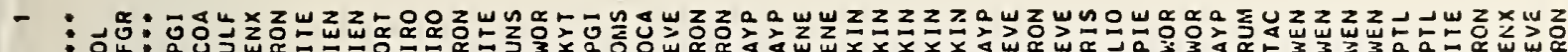

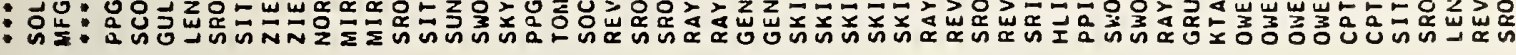

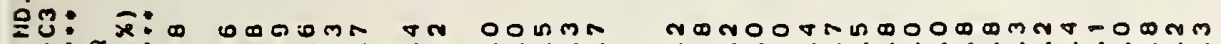
แु

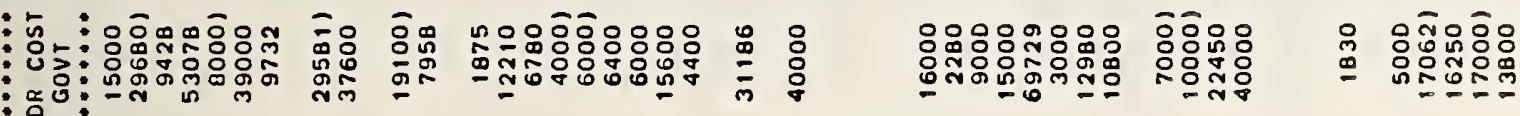

- :

:

:

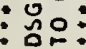

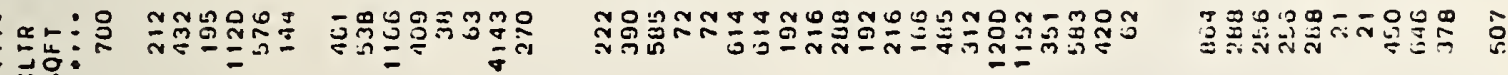
岕宫:

$\ln a:$

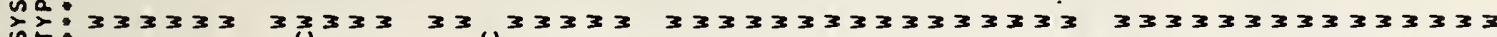
II IIII UIIII U UIIIII IIIIIII IIIIIII IIIIIII IIIII ( 0 :

古:

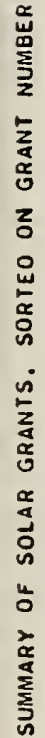

层资:

:

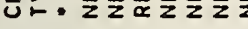

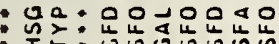

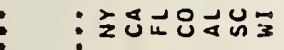

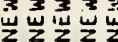

00000

is

잔 五

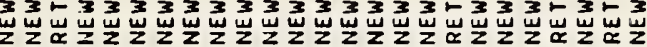

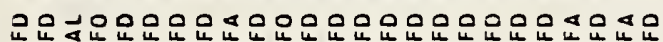

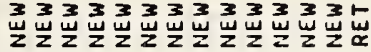

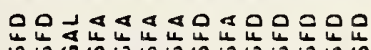

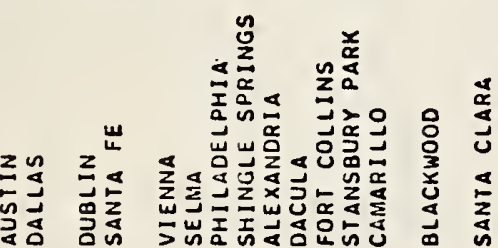

แ:

:

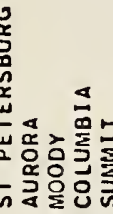
กี

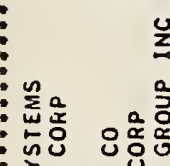

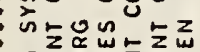

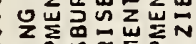

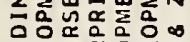

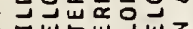

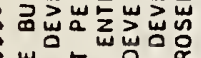

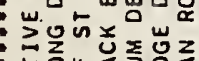

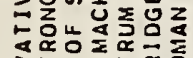

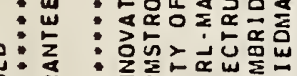

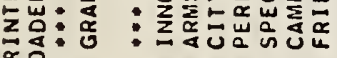

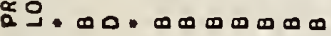
สำ:

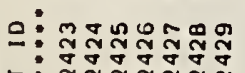

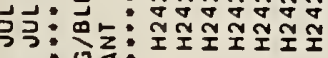

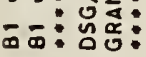

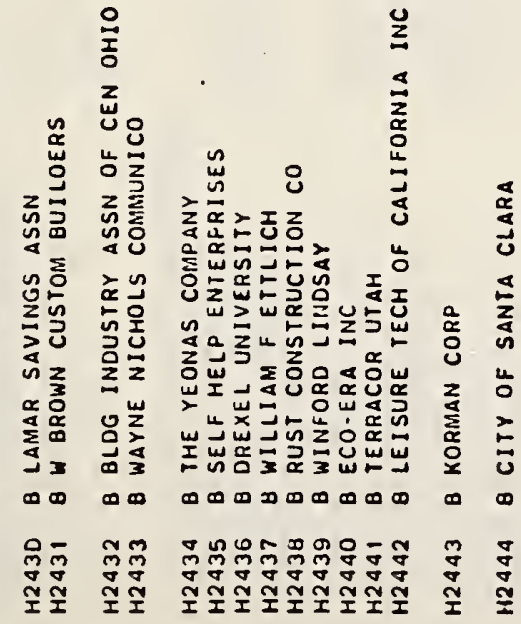

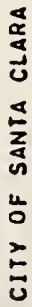

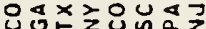

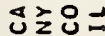

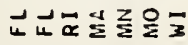

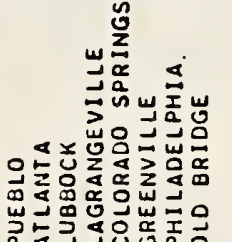

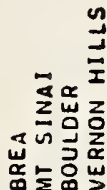

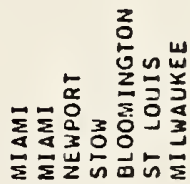

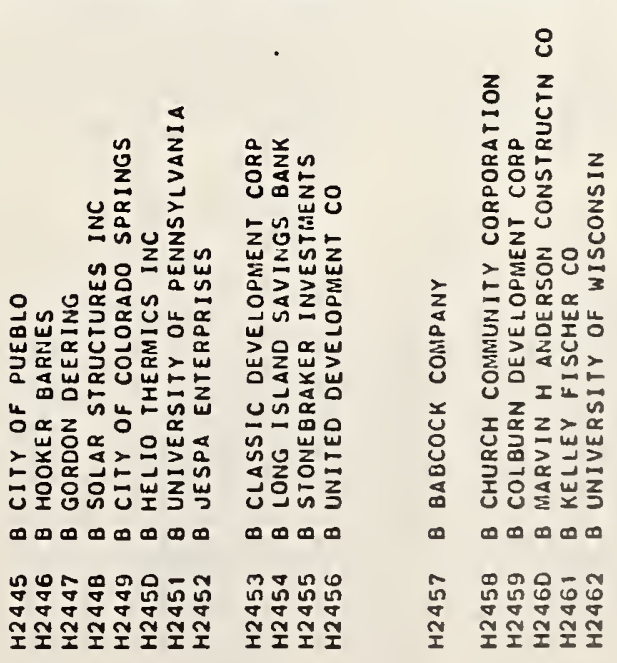


- : : : : 28: 岁它: 若:

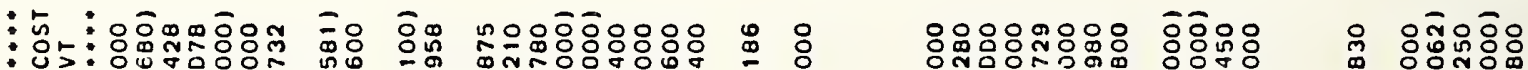

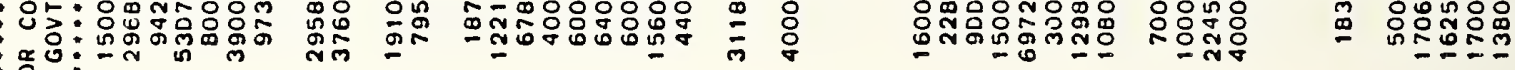

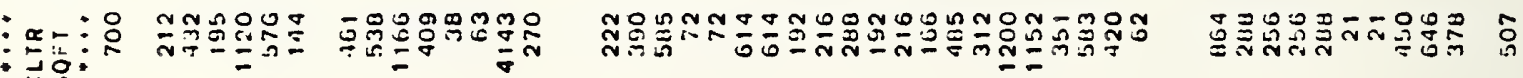
ư

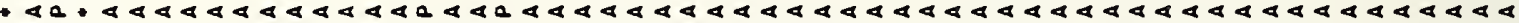

in

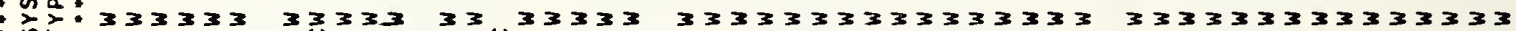
" ñ : II IIII UIIII U U IIIII IIIIIII IIIIIIIIIIIIIII IIIIII

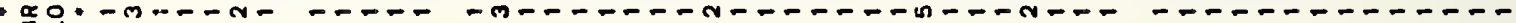
里范:

点

害落:

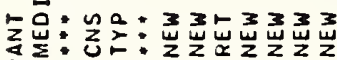

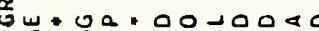

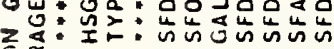

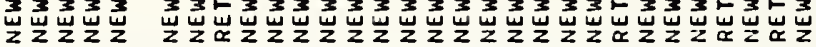

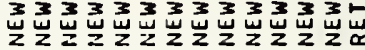
연연연 운은

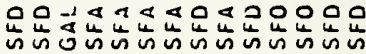

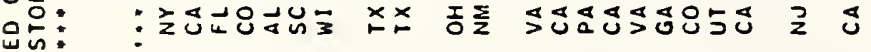

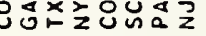

wo:

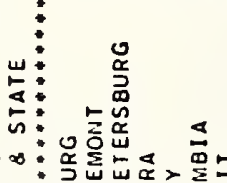

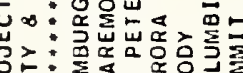
a<smiles>[Si]#[Si]#[Si]</smiles>

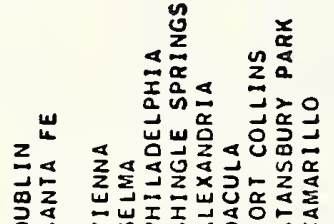

胫: $\vdots \vdots$

空

$\sum^{\bigcup}$

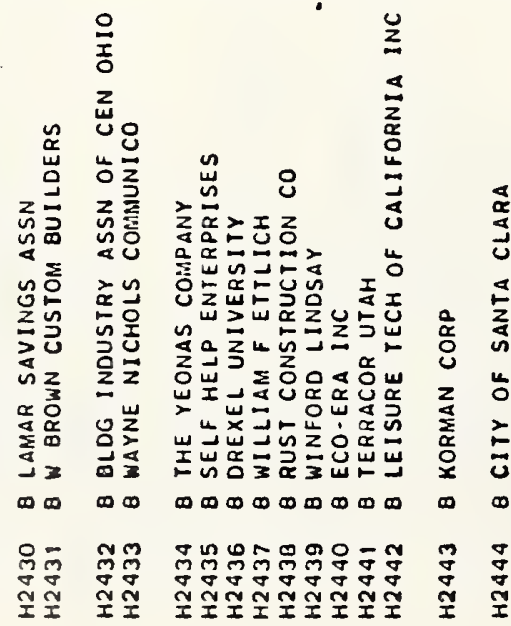

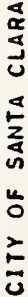

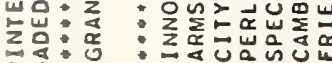

జ

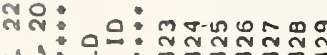

蛋苟:

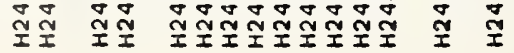

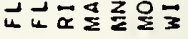
- 

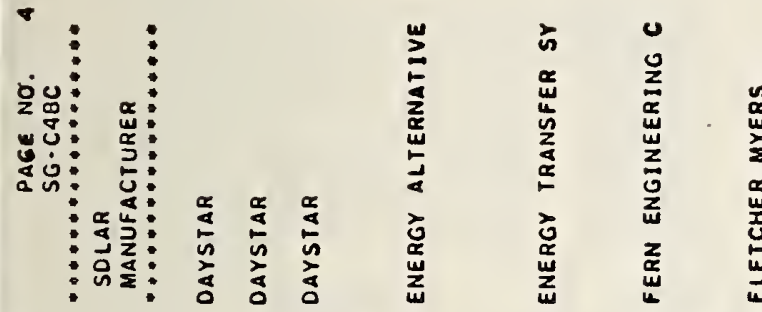

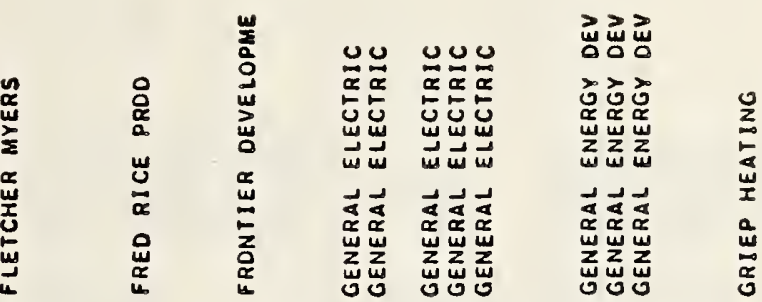

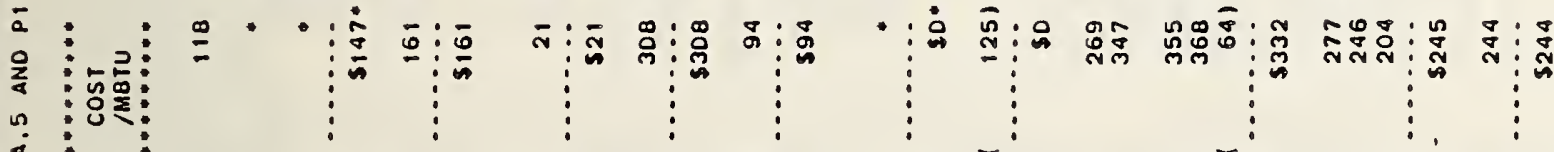

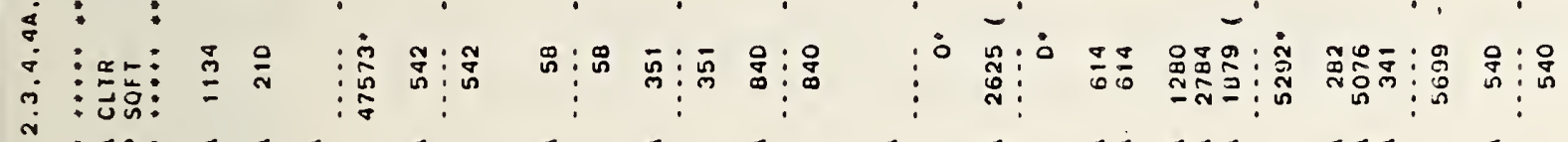

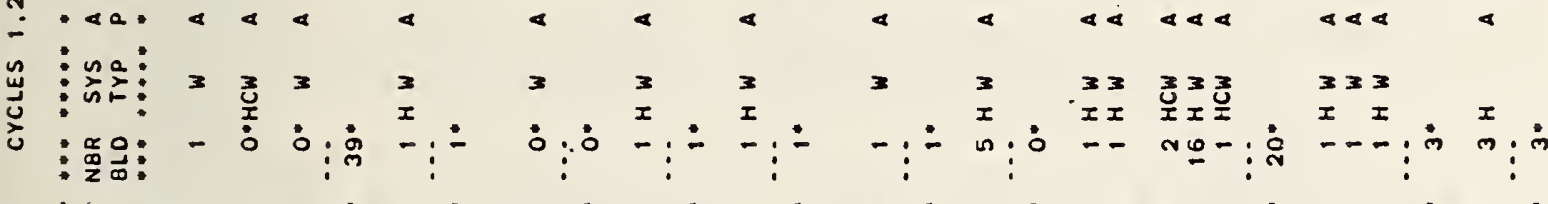

客管: - - -

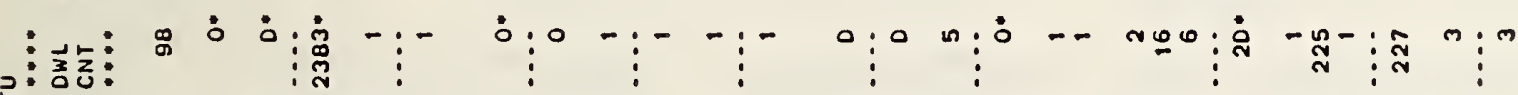

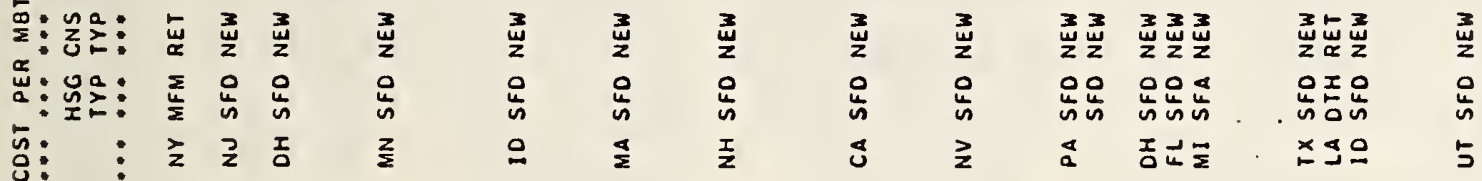

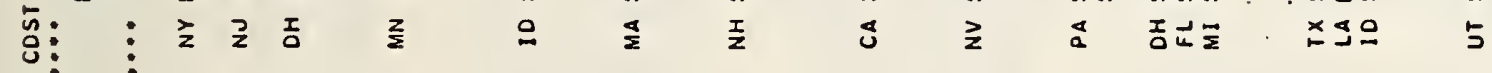

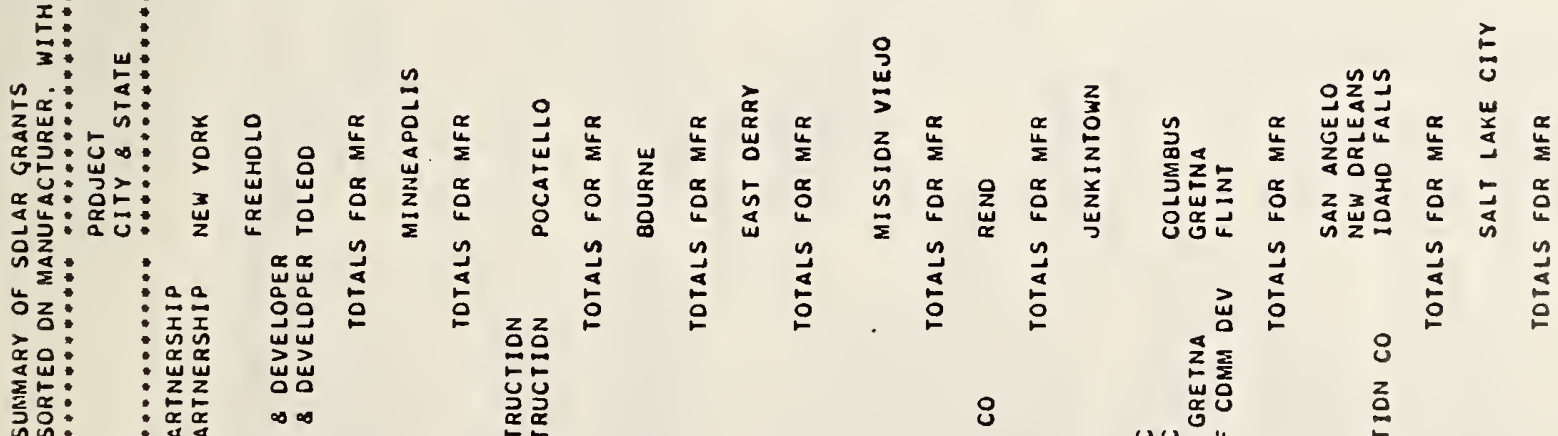

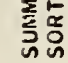

:

$\vdots$

วิ)

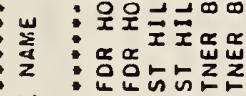

จำ:

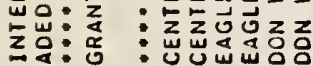

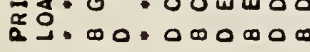

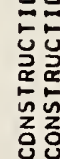

สกละ

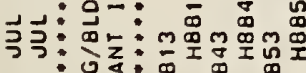

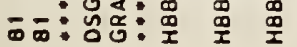
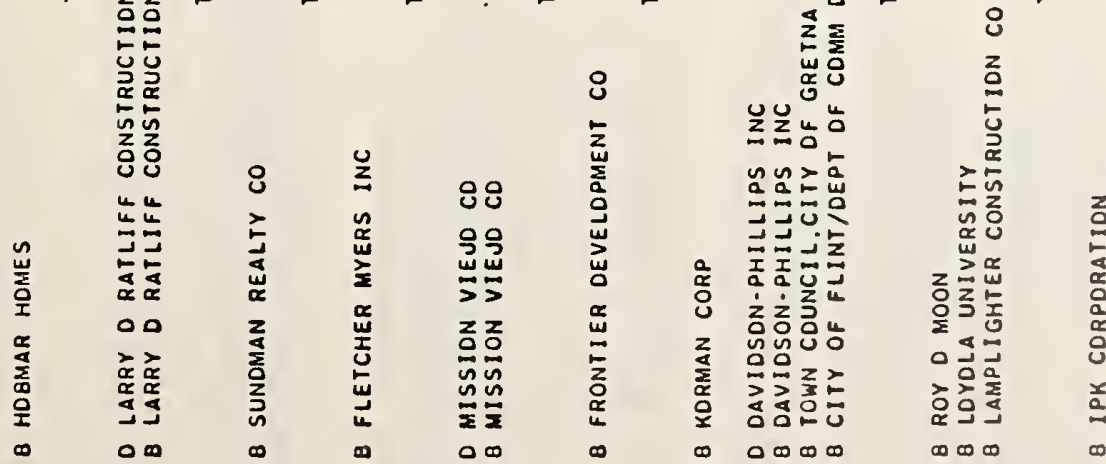

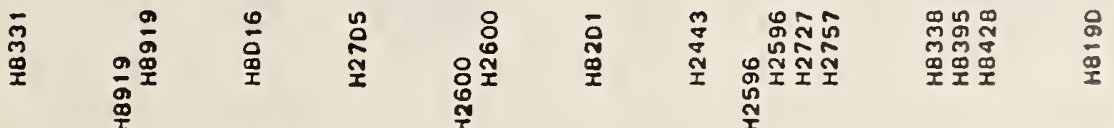




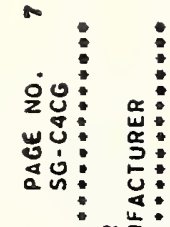

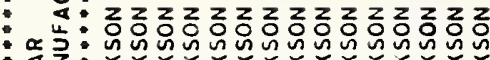

:

а :

ก : 50

:

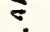

(2)

I

:

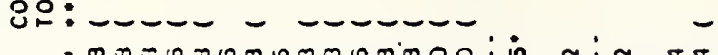
起总:

140.444444444444444

ina:

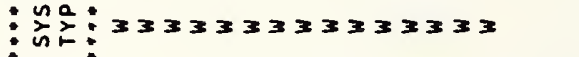

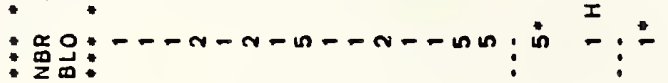

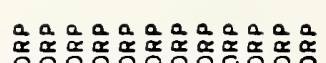

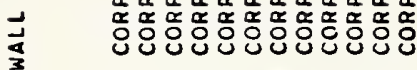

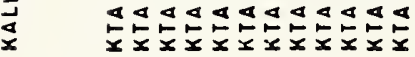

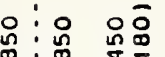

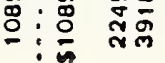

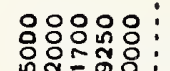

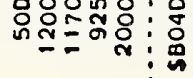

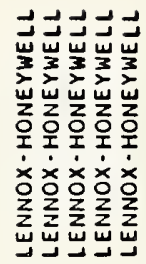

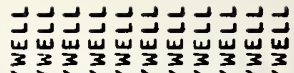

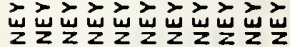

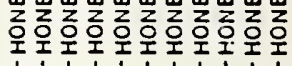

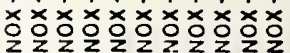

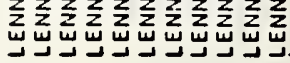

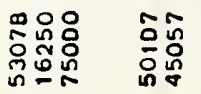

ตํํํำำกำ

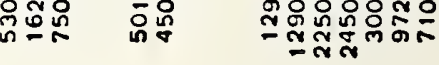

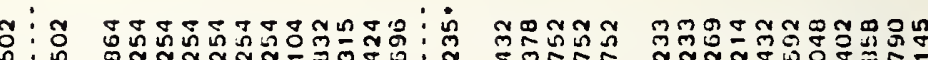

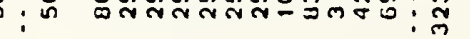

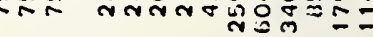

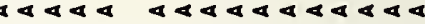

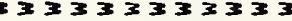

IIIIIII III

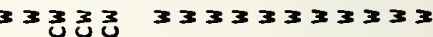

II논인IIII

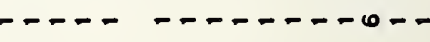

英公:

势:

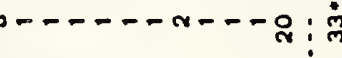

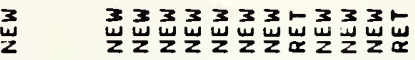

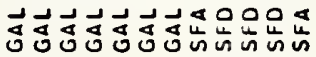

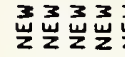
00000

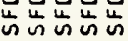

운존

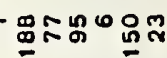

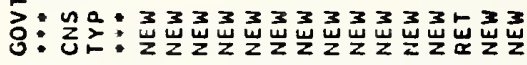

운

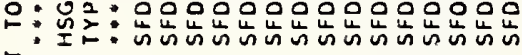

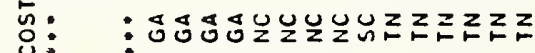

$a$

舷

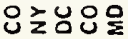

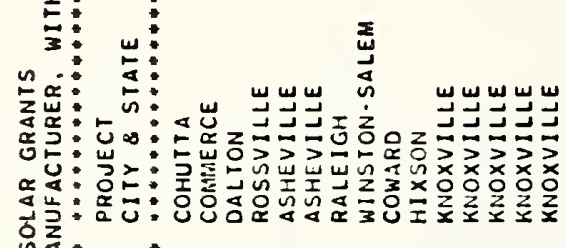

家:

능

究是

$\vdots$

U

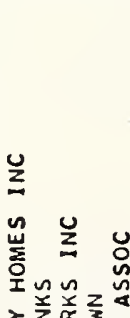

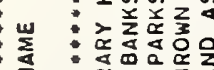

w :

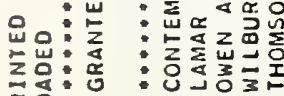

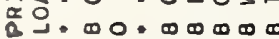

원음

工㐫

号음

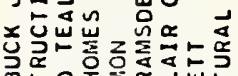

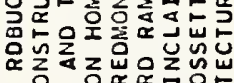

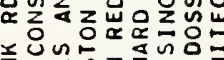

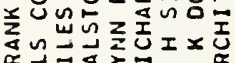

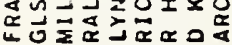

สิก

$\infty \infty \infty \infty \infty \infty \infty \infty)$

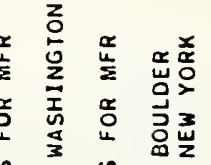

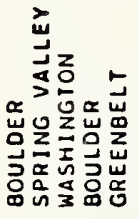

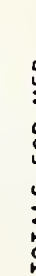

중음

这运要

的了品证

岁岕

送

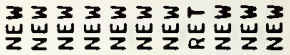

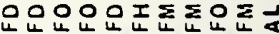

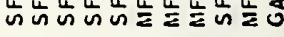

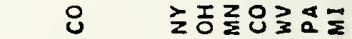

造

点.

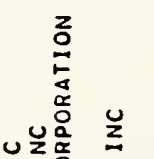

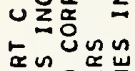

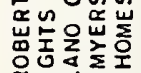

凯岮

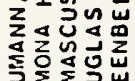

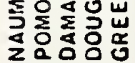

$\infty \infty 0 \infty$

崩家

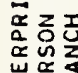

88

i

웄욿

허믐

$\sum_{i=2}$

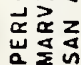

증름

药药

$\infty \infty$

OD

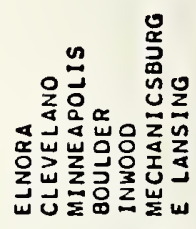

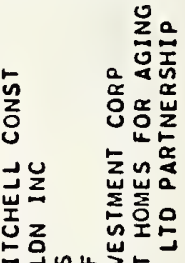

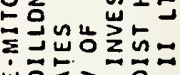
它目己

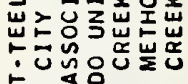

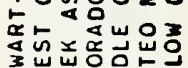

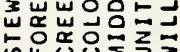

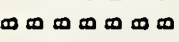

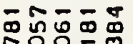

节节:

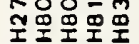

ํํ융

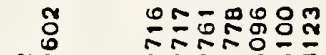

꼬꼬ำ

ํํำ

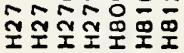
- 


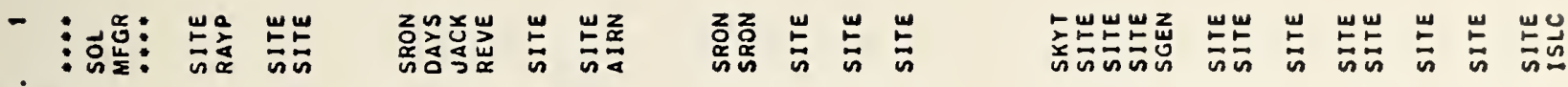

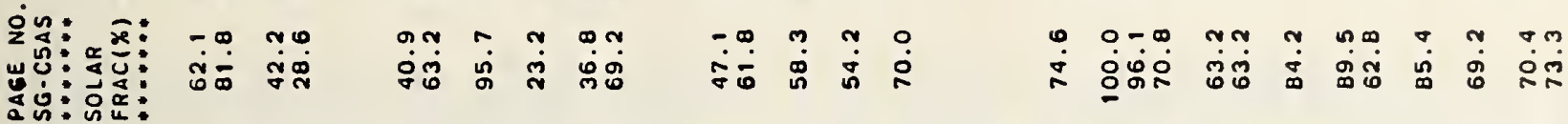

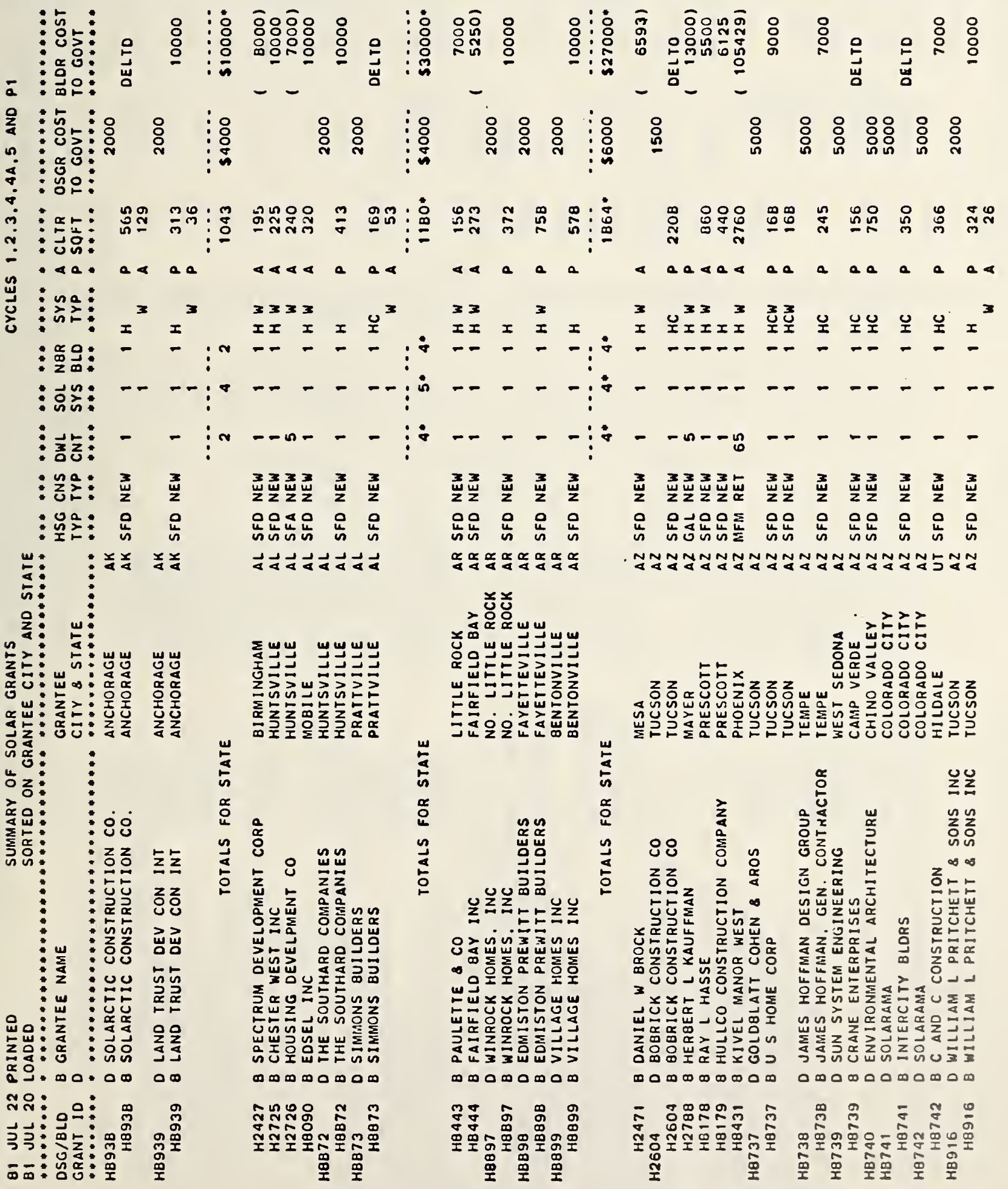




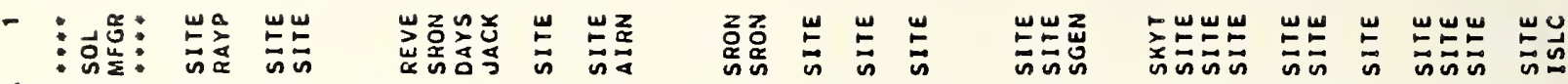

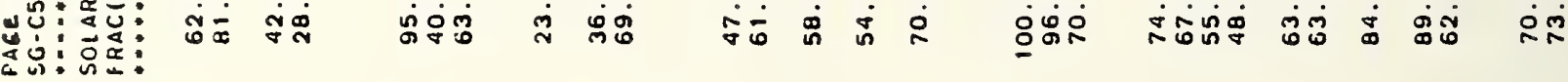

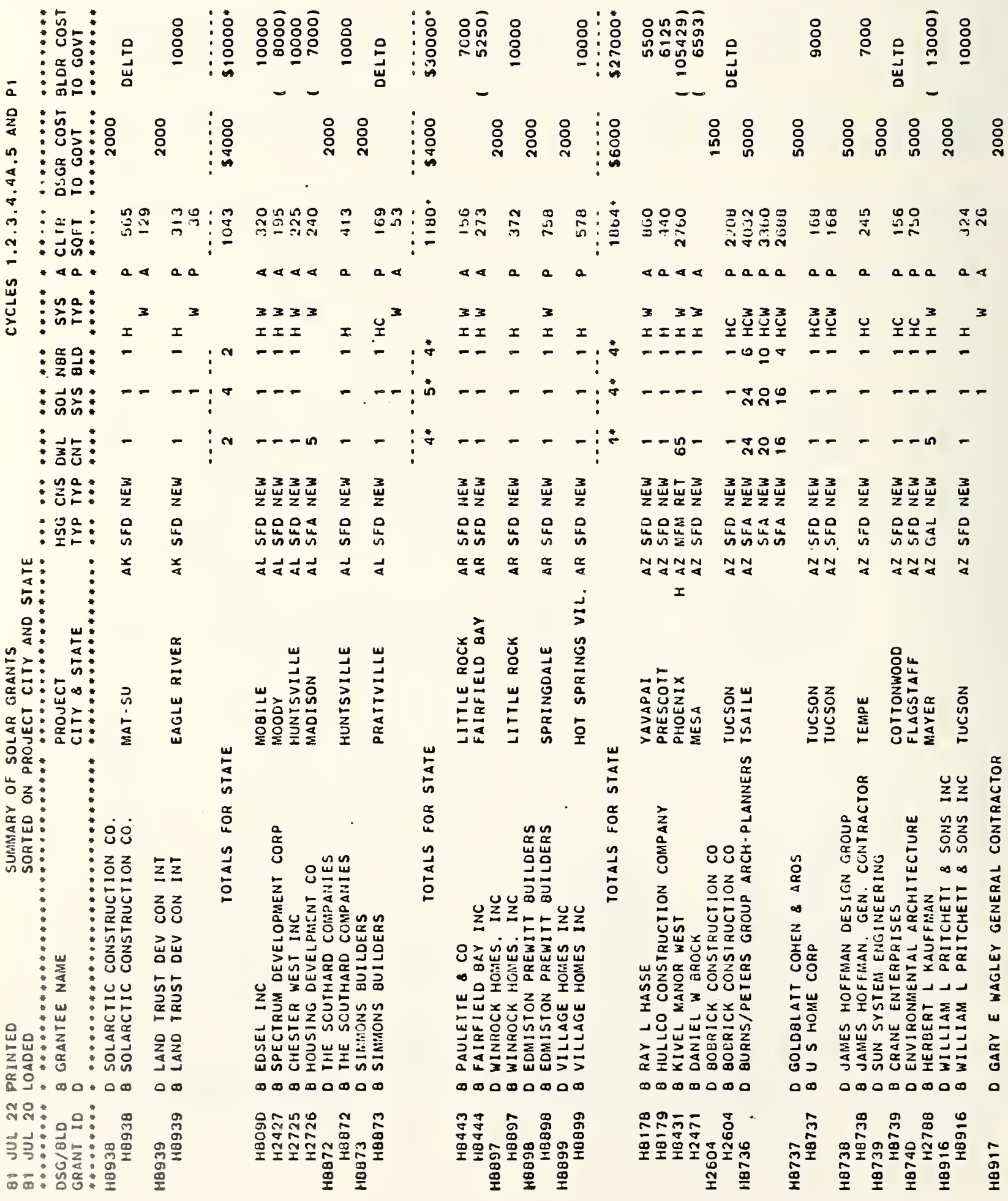




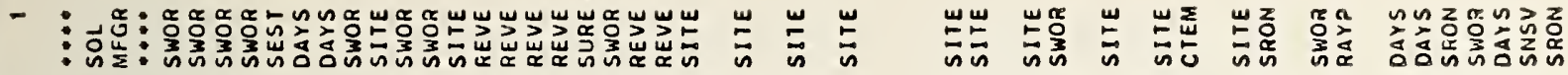

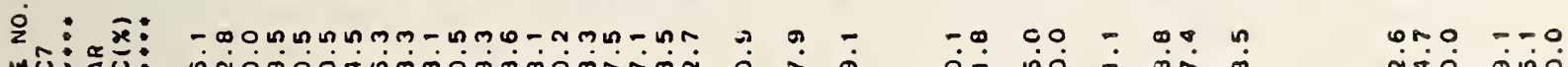

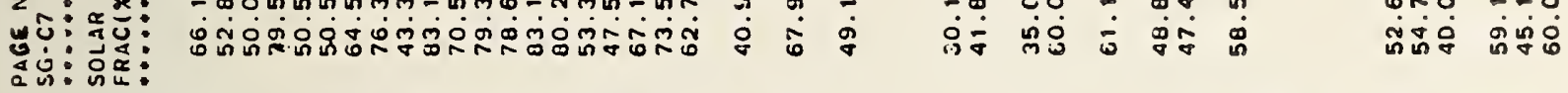

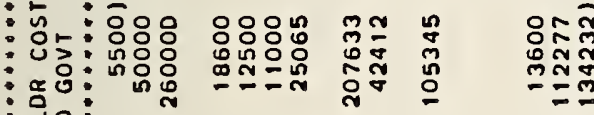

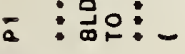

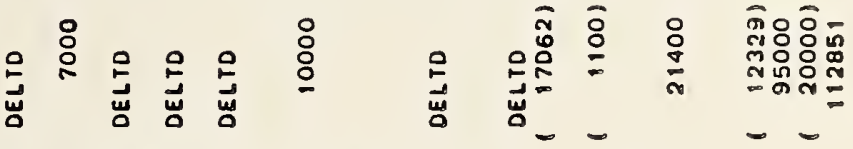

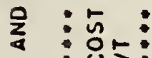

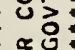

:

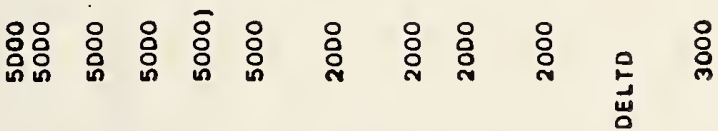

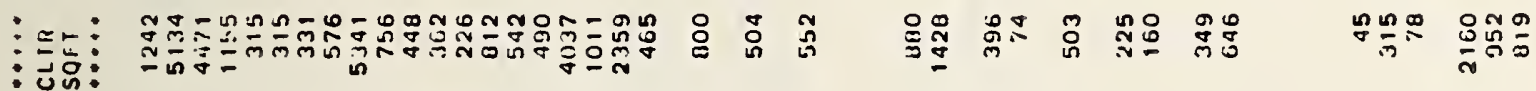

-

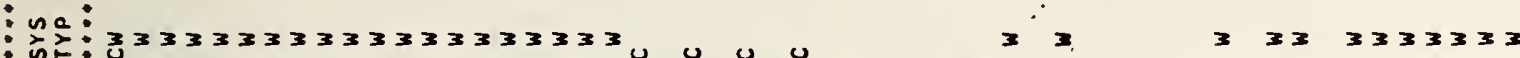

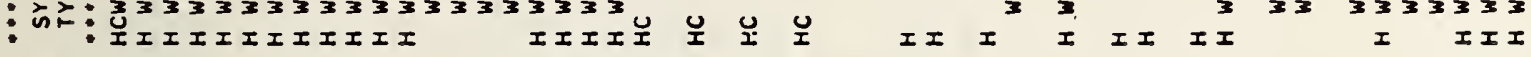
:

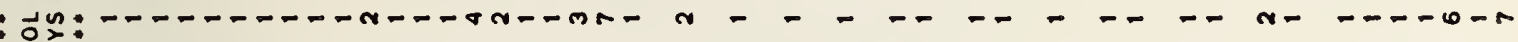

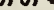

:

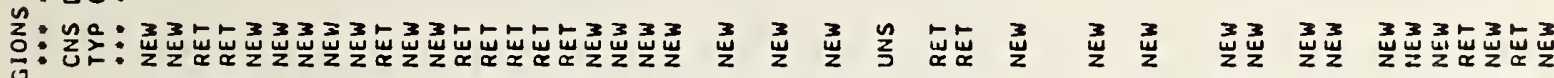

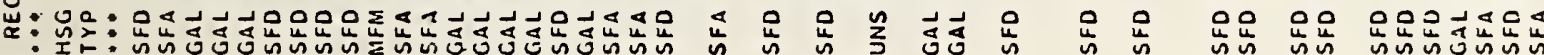

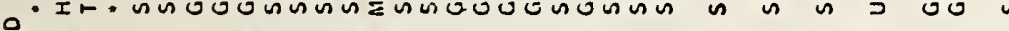

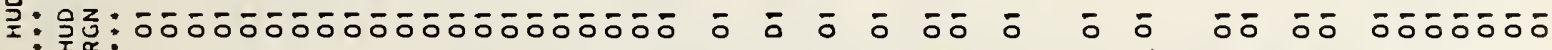

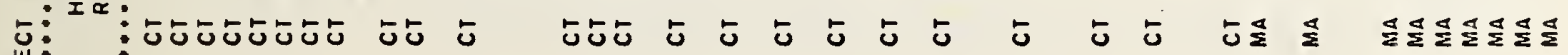

na.

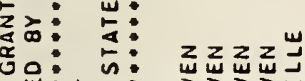

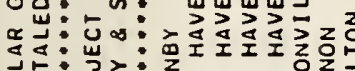

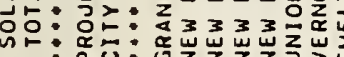
눙은

空空:
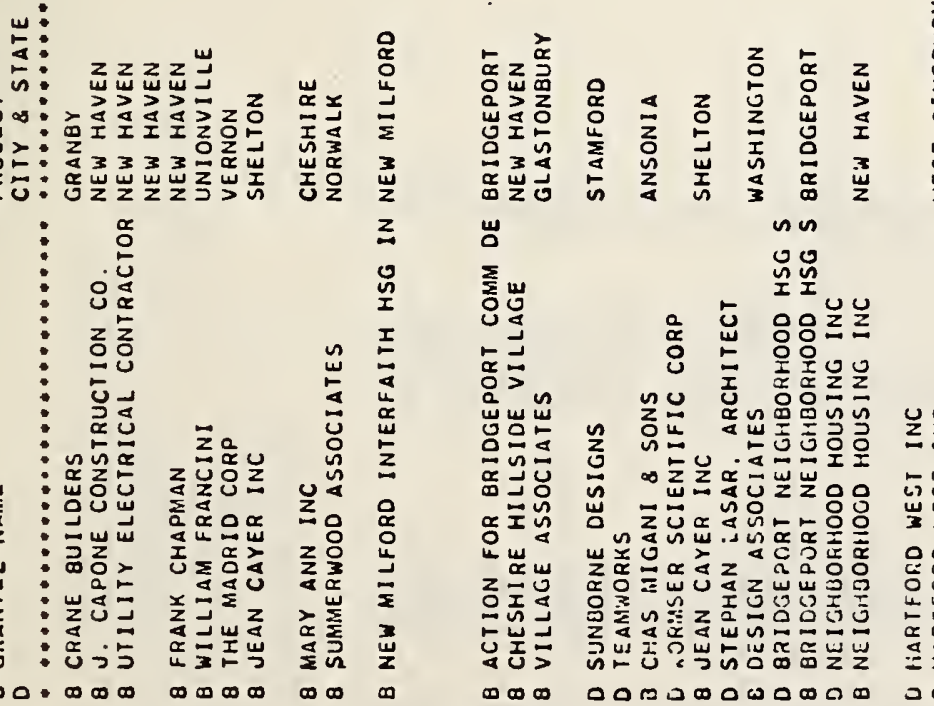

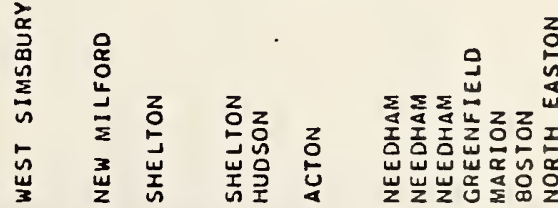

茨

홍ํㅓㄴ

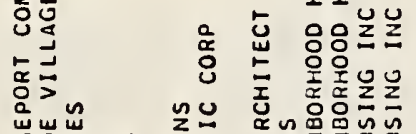

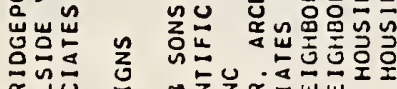

通颃

퐁

空出

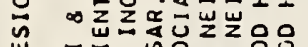

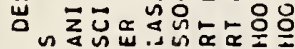

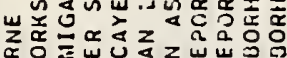

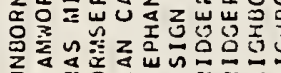

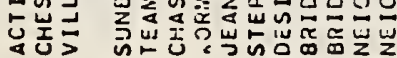

$\infty \omega$

DOMDOONODOD
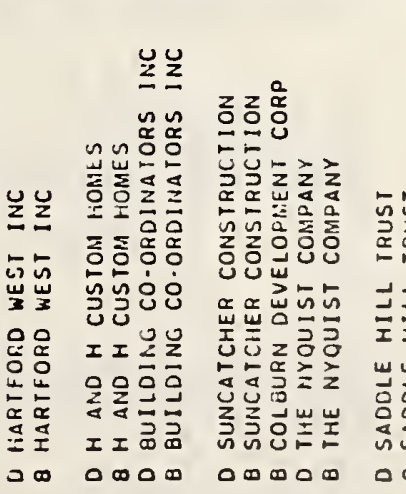

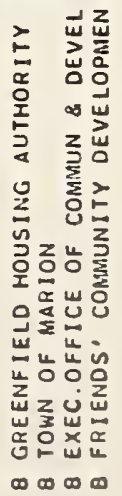

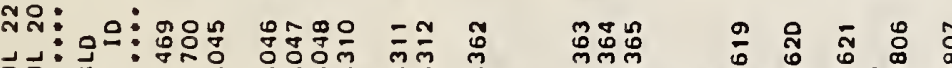

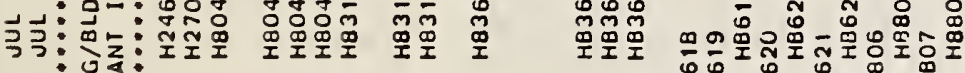

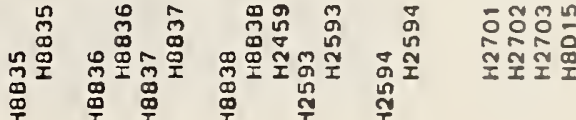

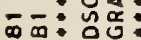

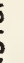

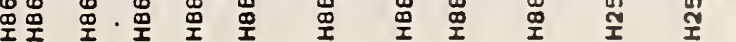




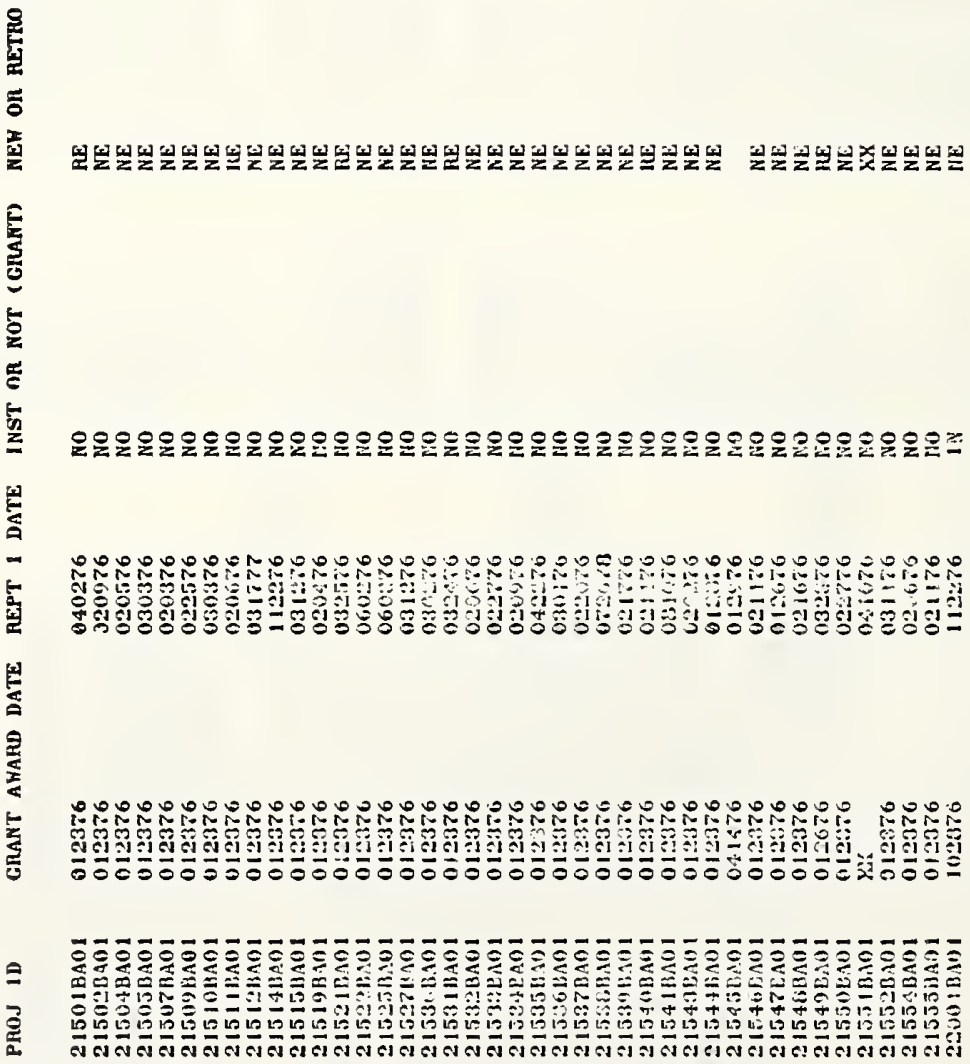



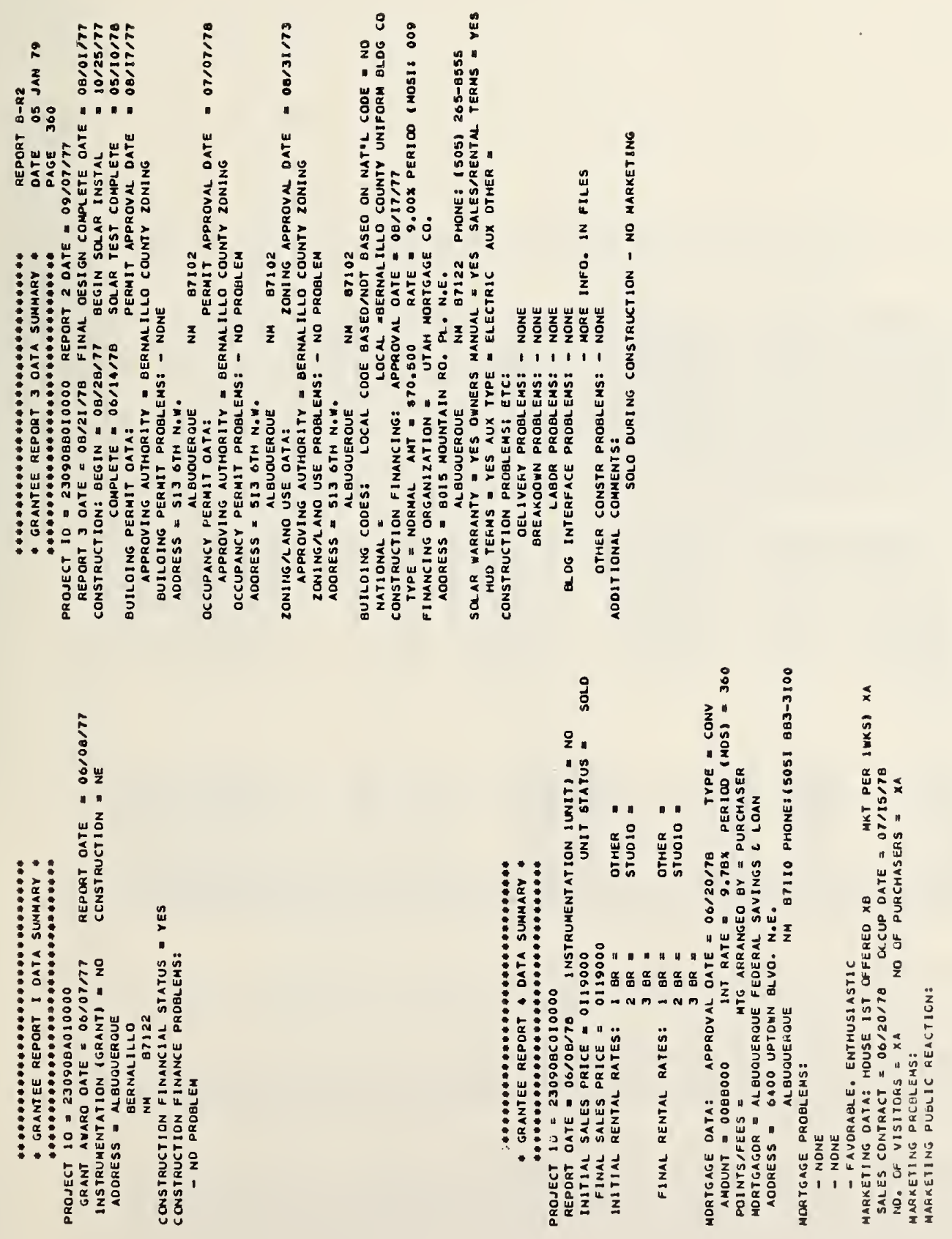


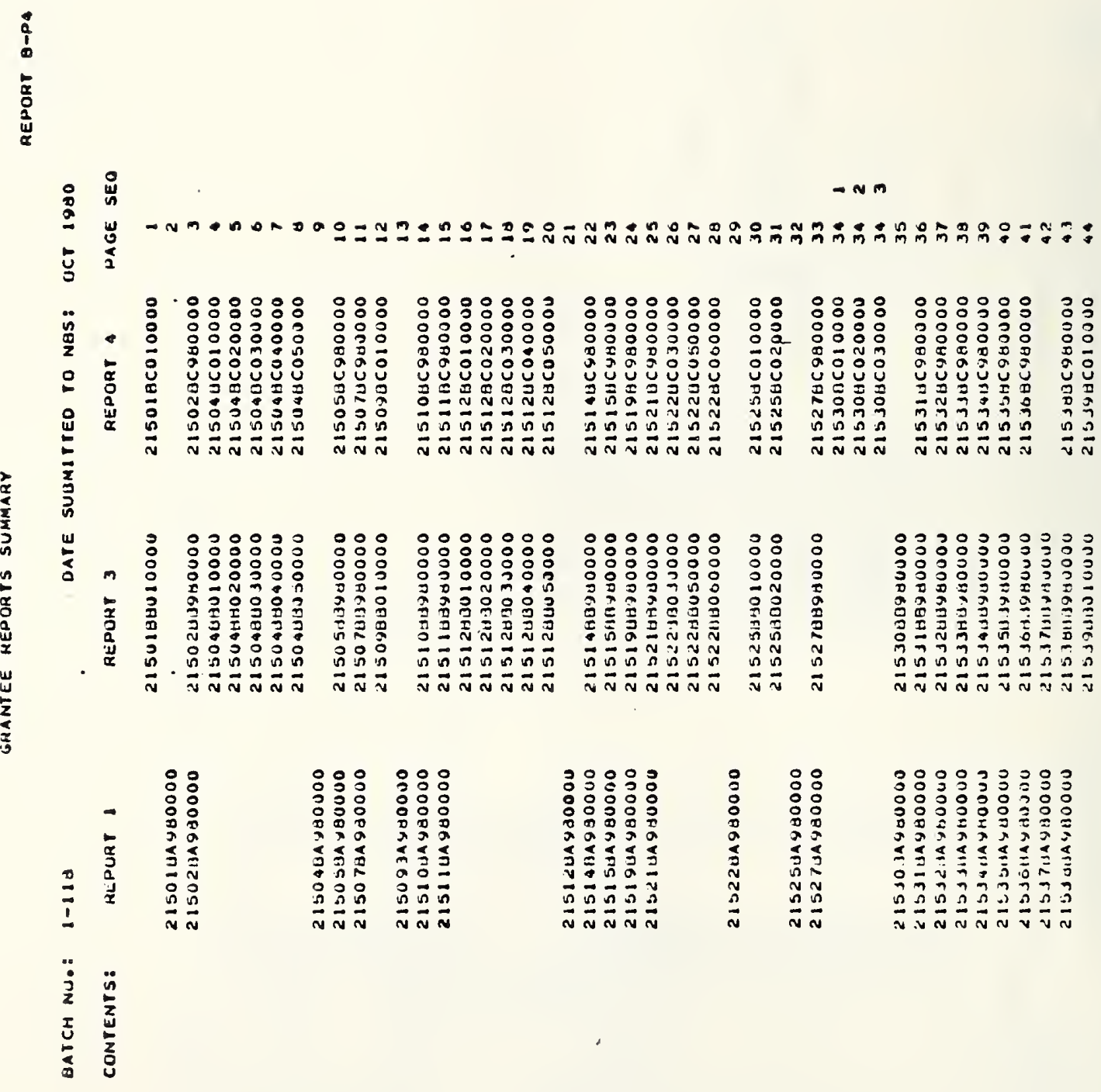




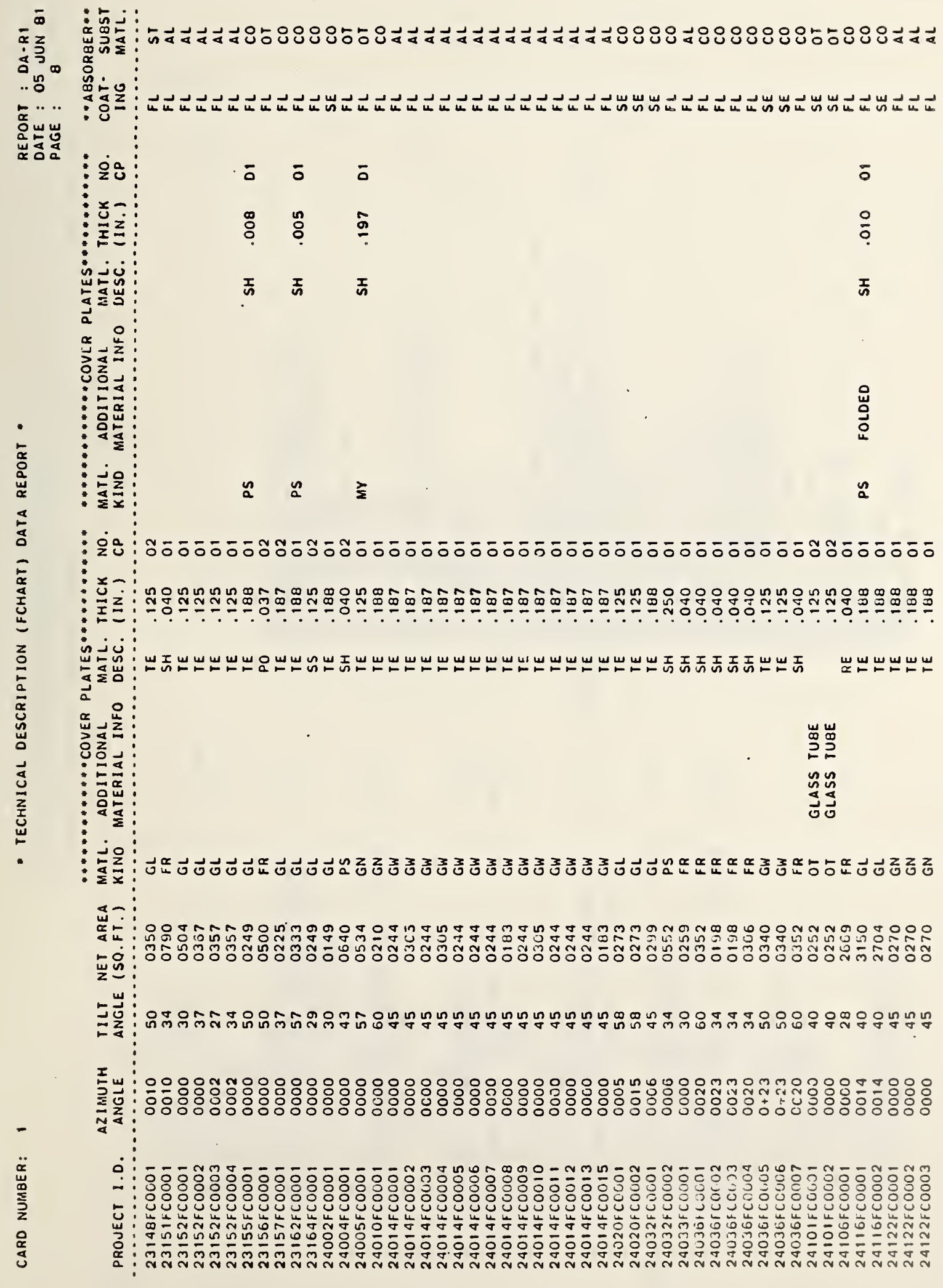



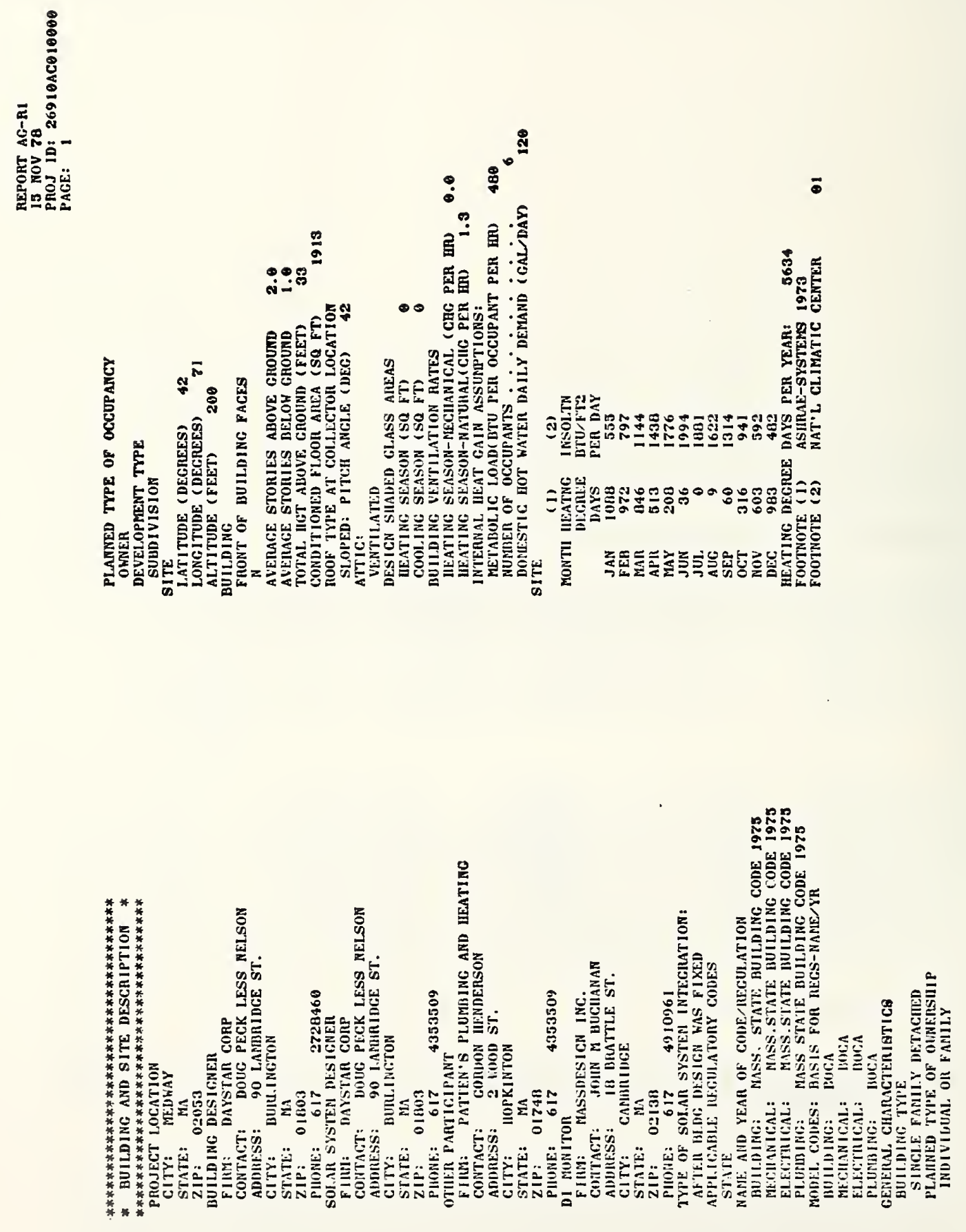


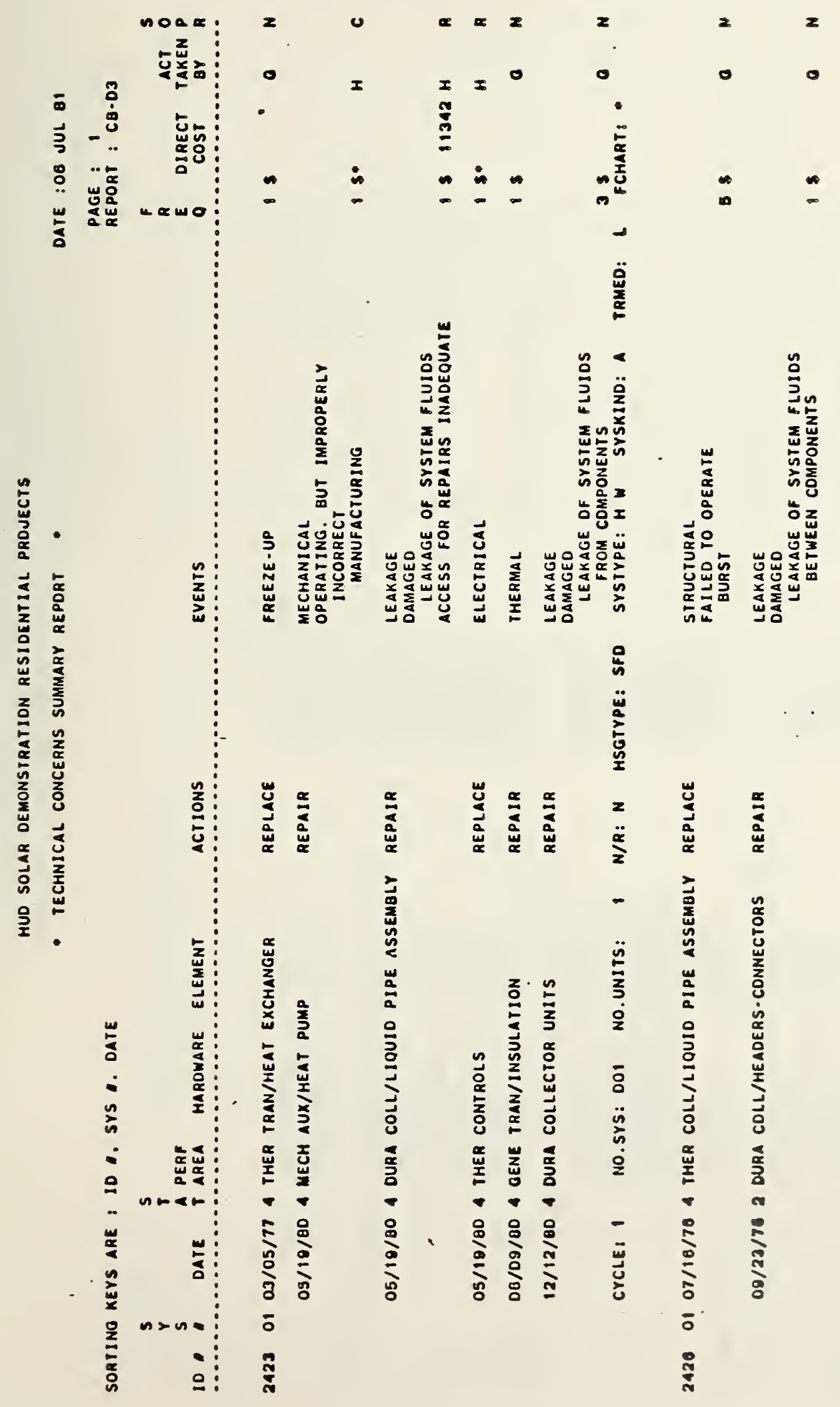




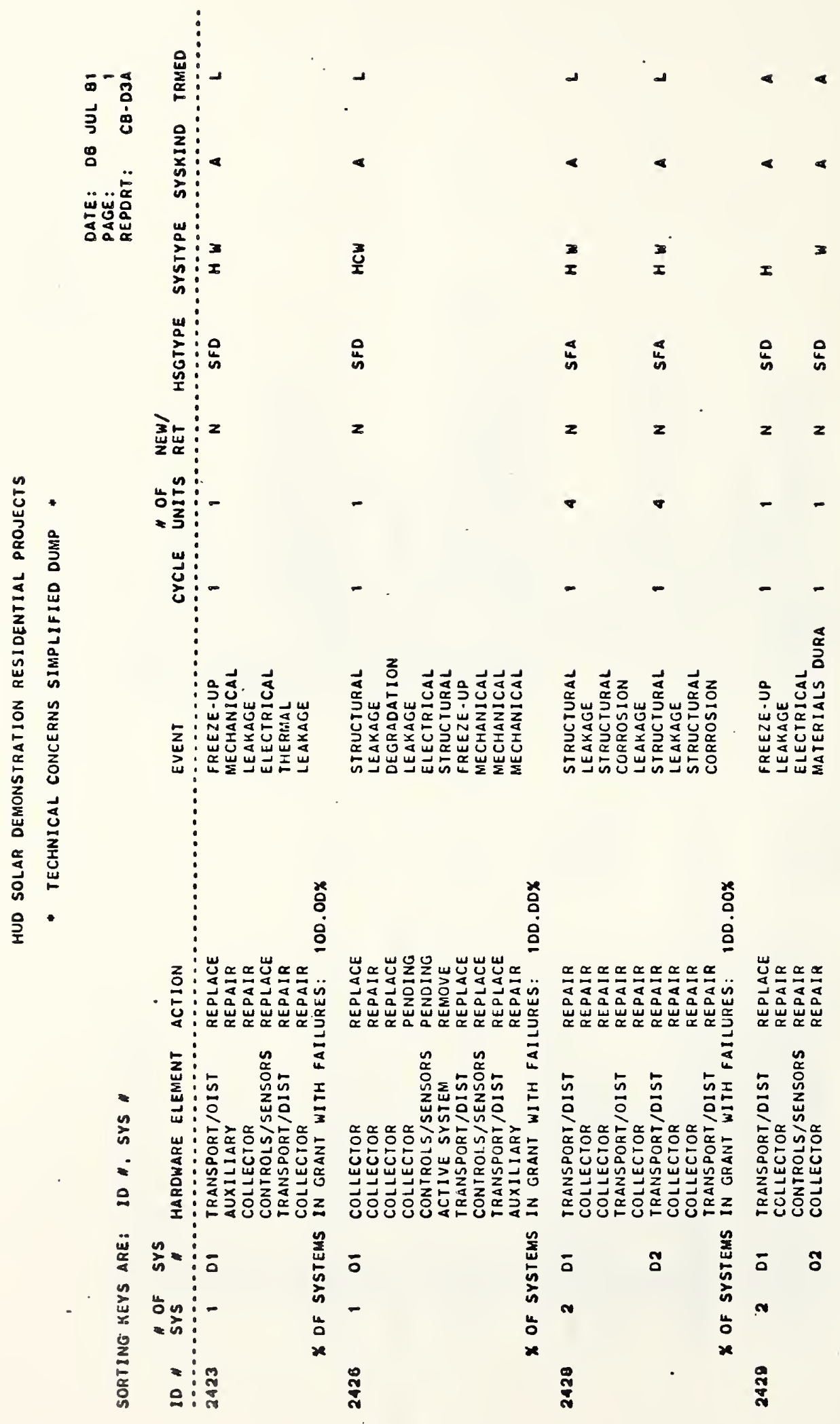




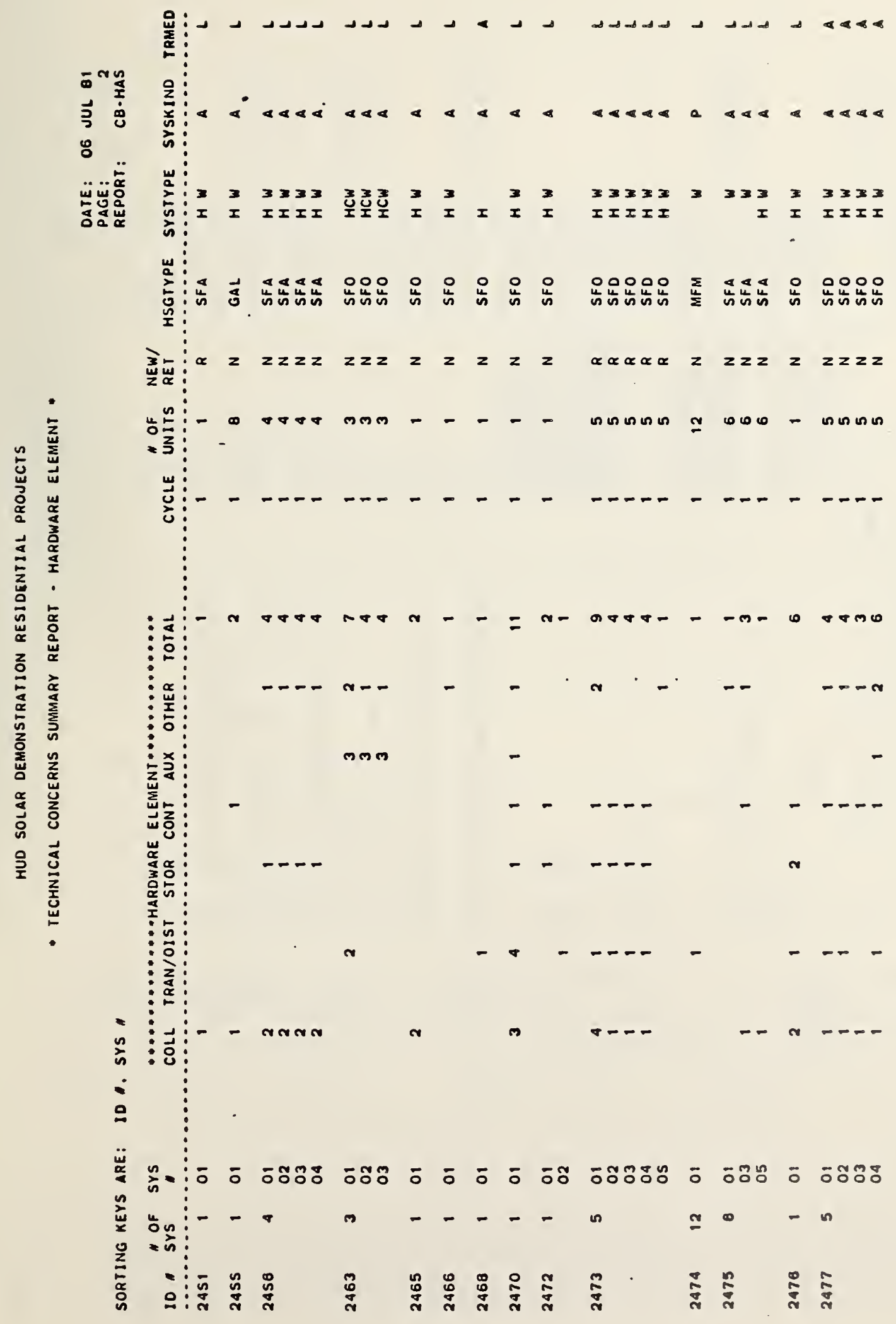




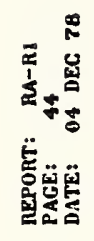

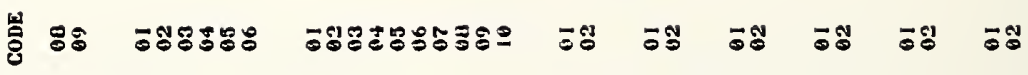

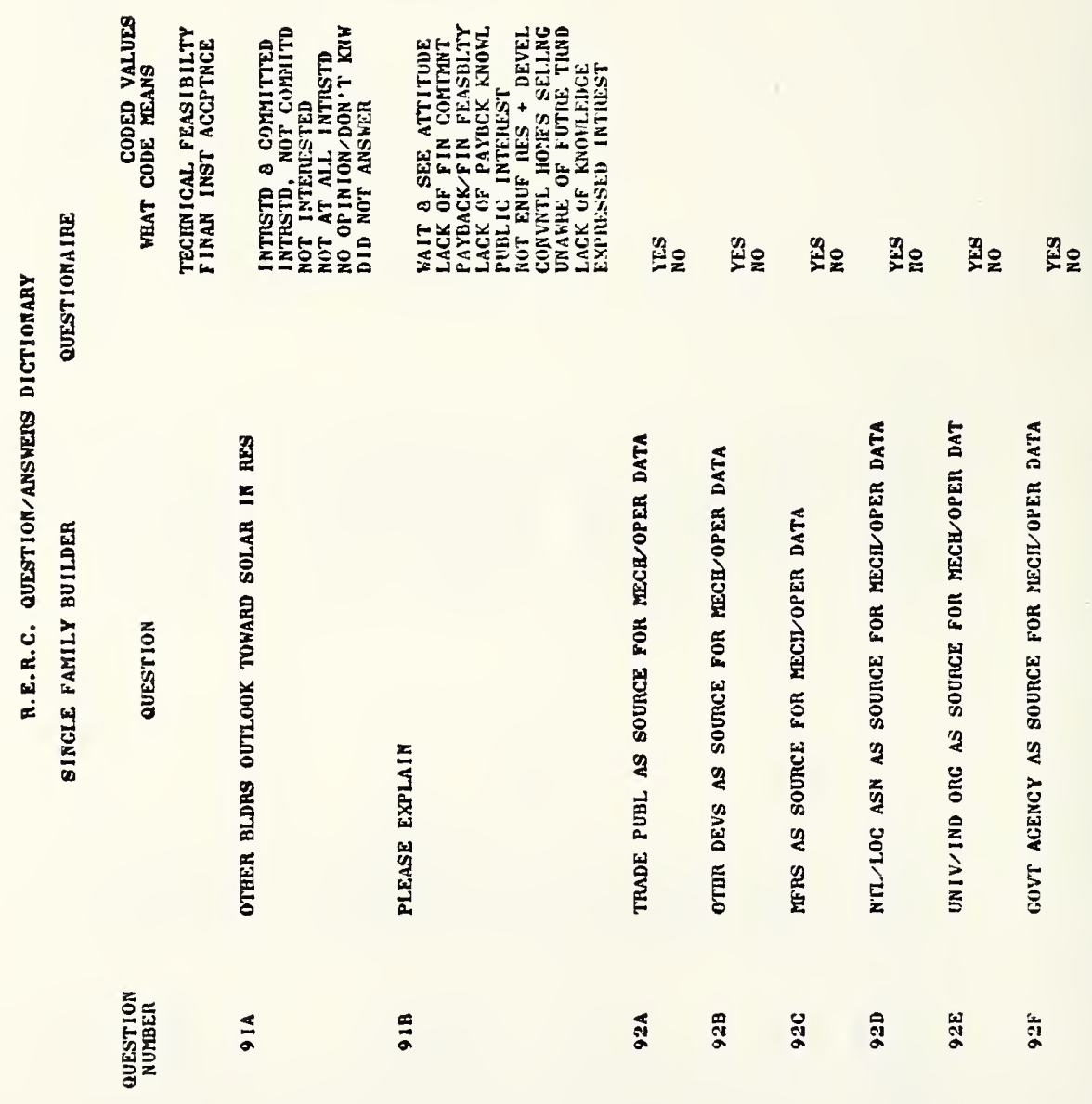




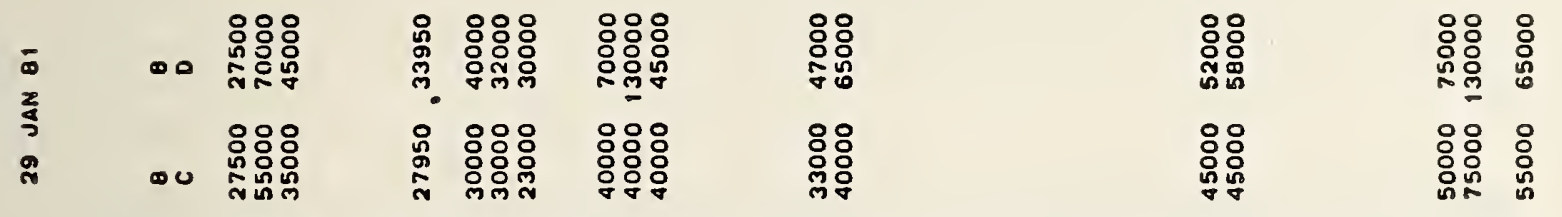

$\infty$

$\infty<$

$0 x$

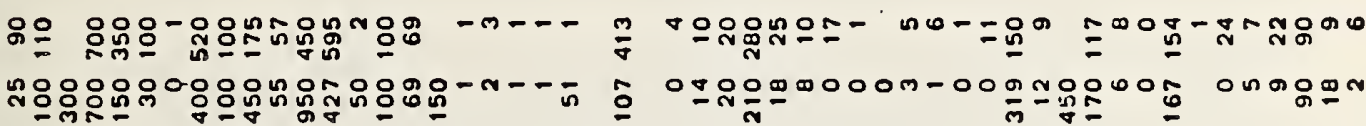

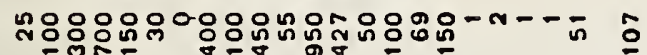

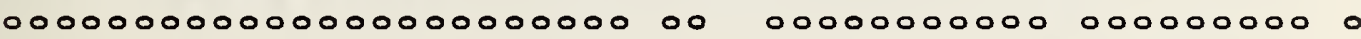
00000000000000000000000000000000000000000000000 00000000000000000000008000000000000000000000000 00000000000000000000000000000000000000000000000 00000008000000000000008000000000000000 N000000000

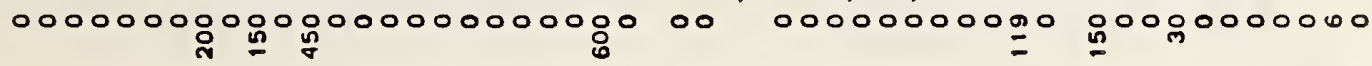
พูก

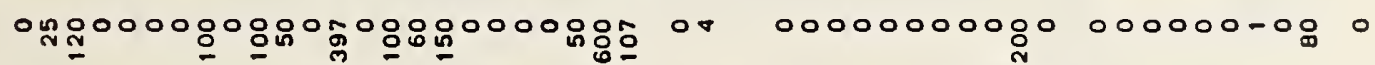

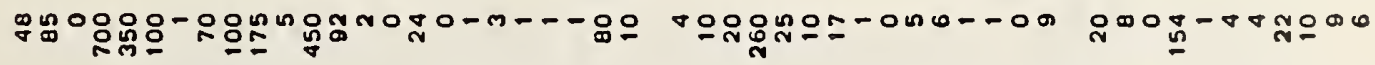

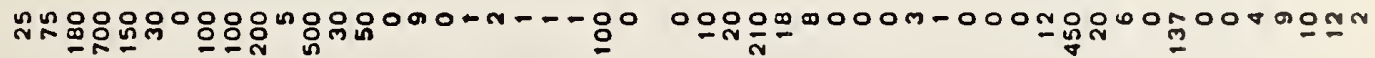

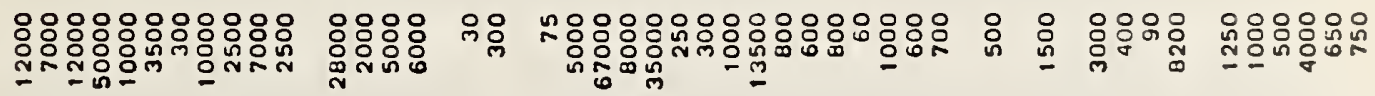

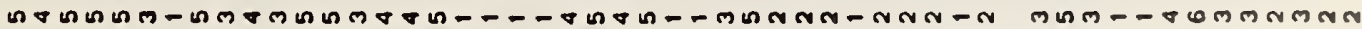

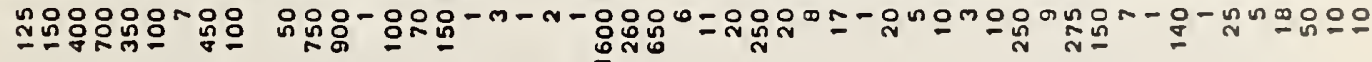

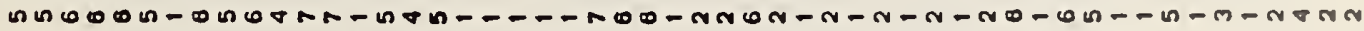

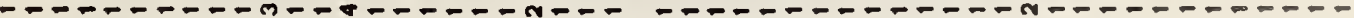
xmจ

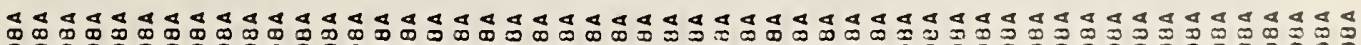

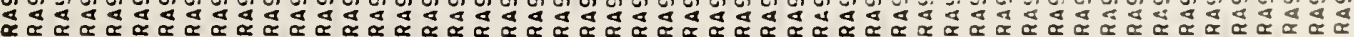

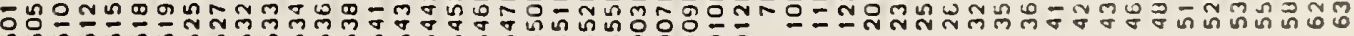

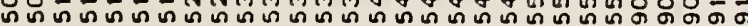

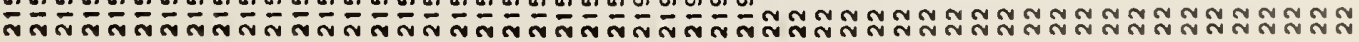




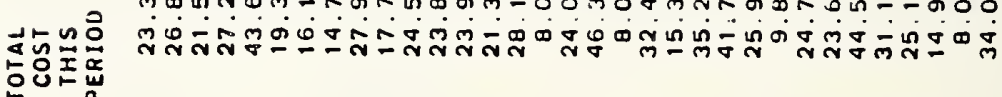

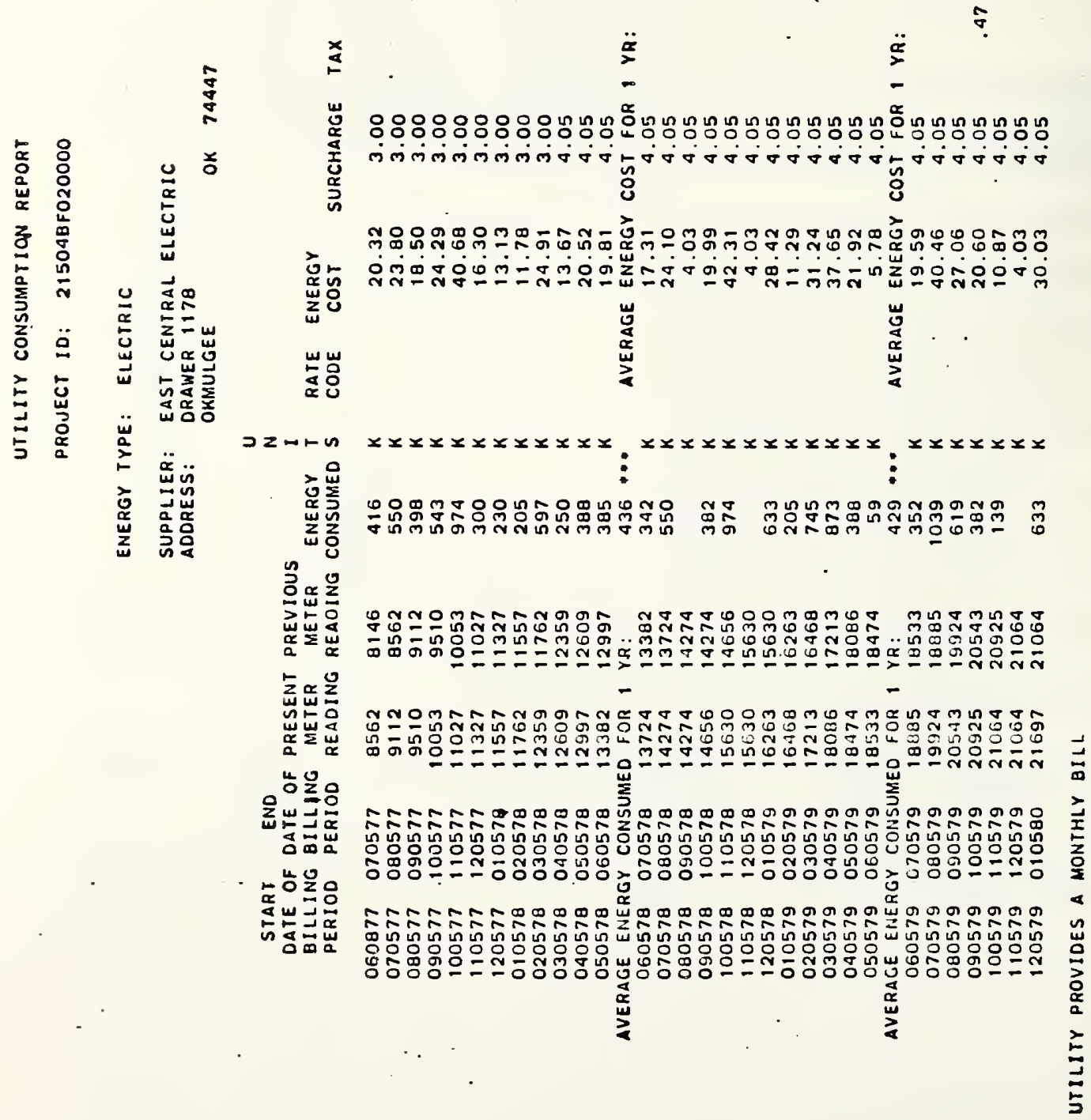




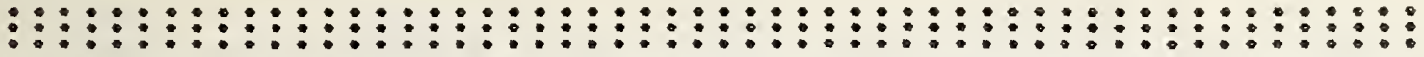

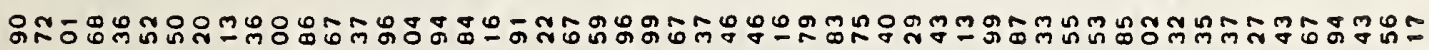

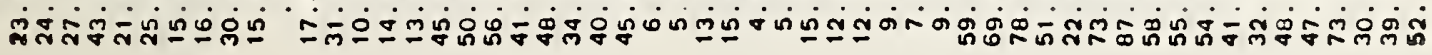

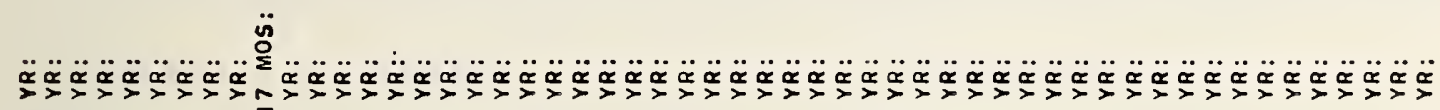

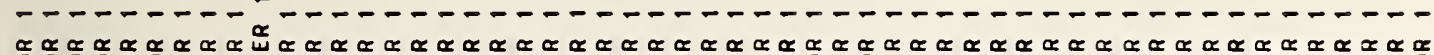

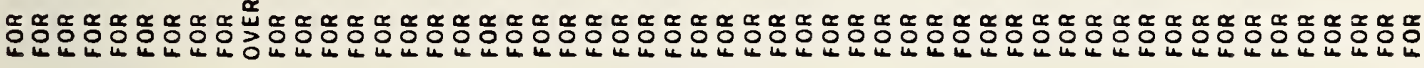

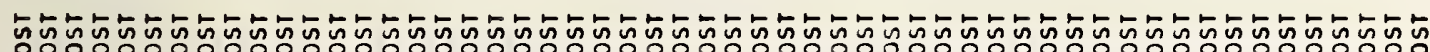

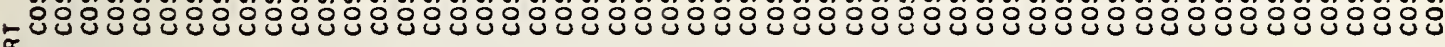

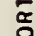

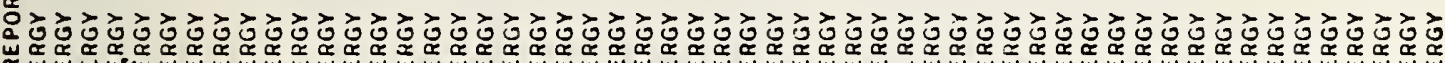

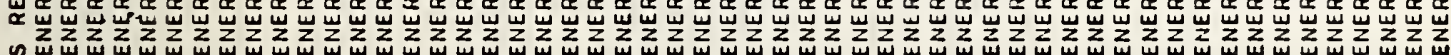
لبّ

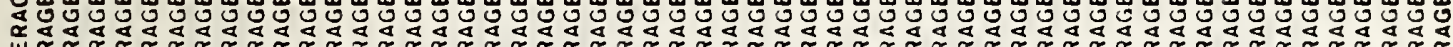

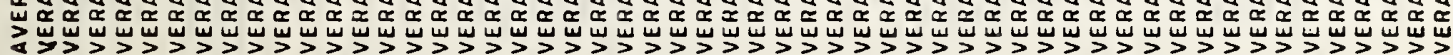
zo

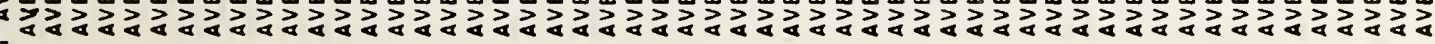

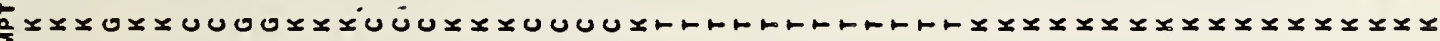
今

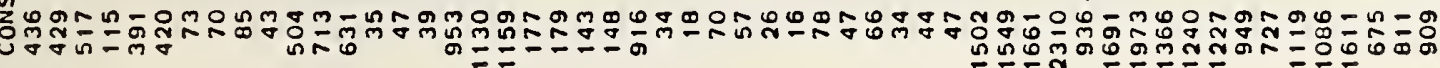


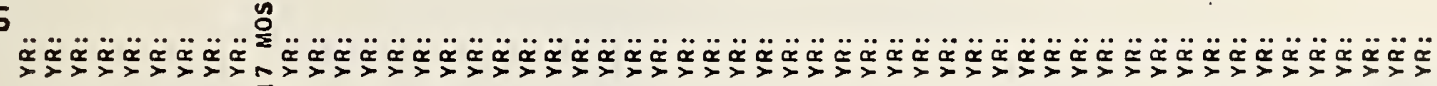
(1)

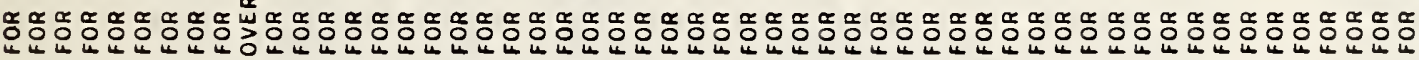

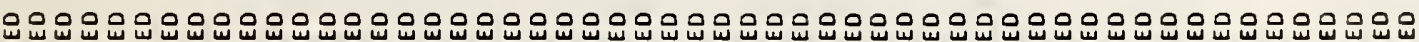

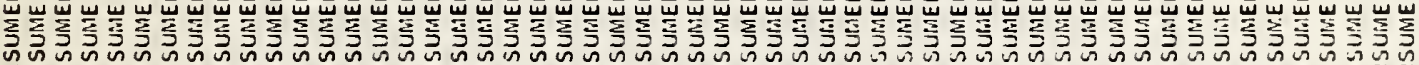

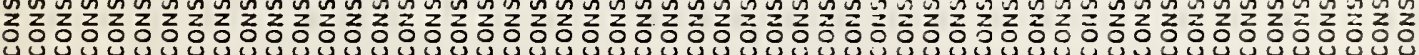

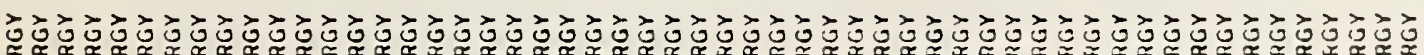

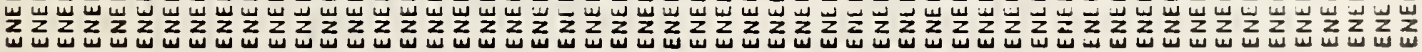

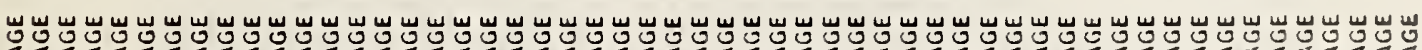

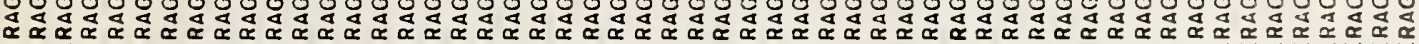

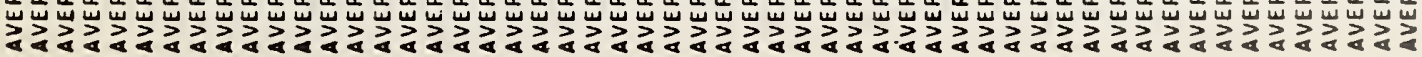

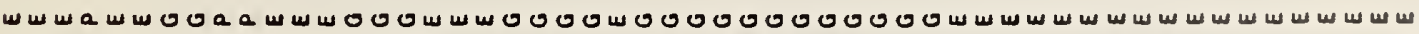
ㅇㅇㅇㅇㅇㅇㅇㅇㅇㅇㅇㅇㅇㅇㅇㅇㅇㅇㅇㅇㅇㅇㅇㅇㅇㅇㅇㅇㅇㅇㅇㅇㅇㅇㅇㅇㅇㅇㅇㅇㅇㅇㅇㅇㅇㅇㅇㅇㅇㅇㅇㅇㅇㅇㅇㅇㅇㅇㅇㅇㅇ

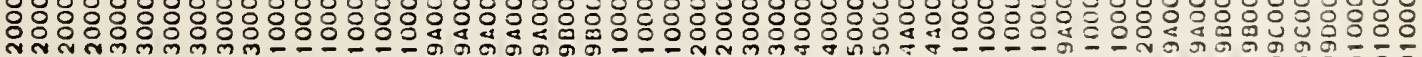

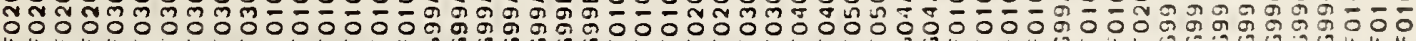

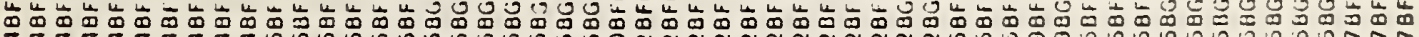

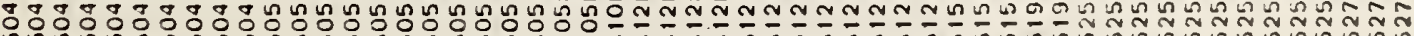

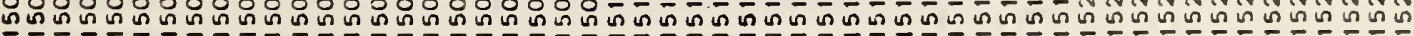
ส 


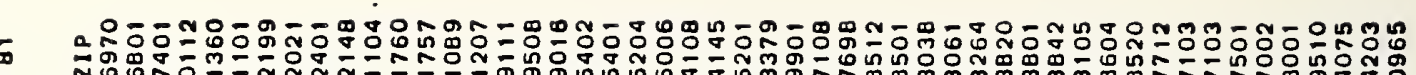

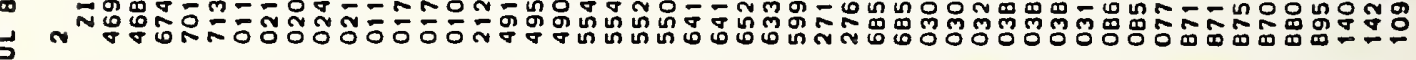

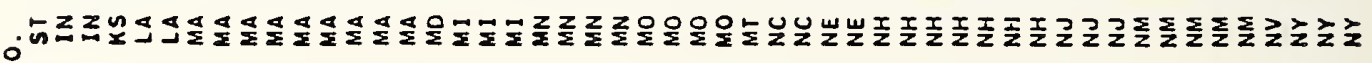

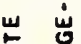

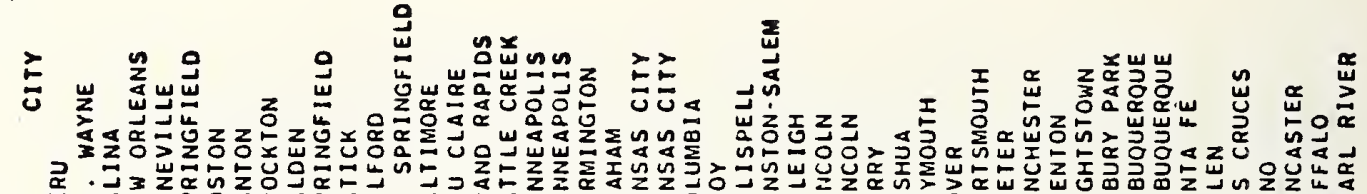

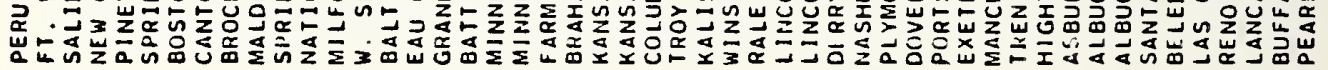

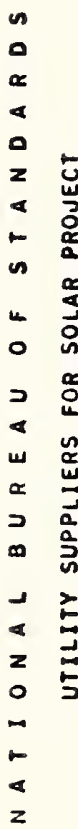
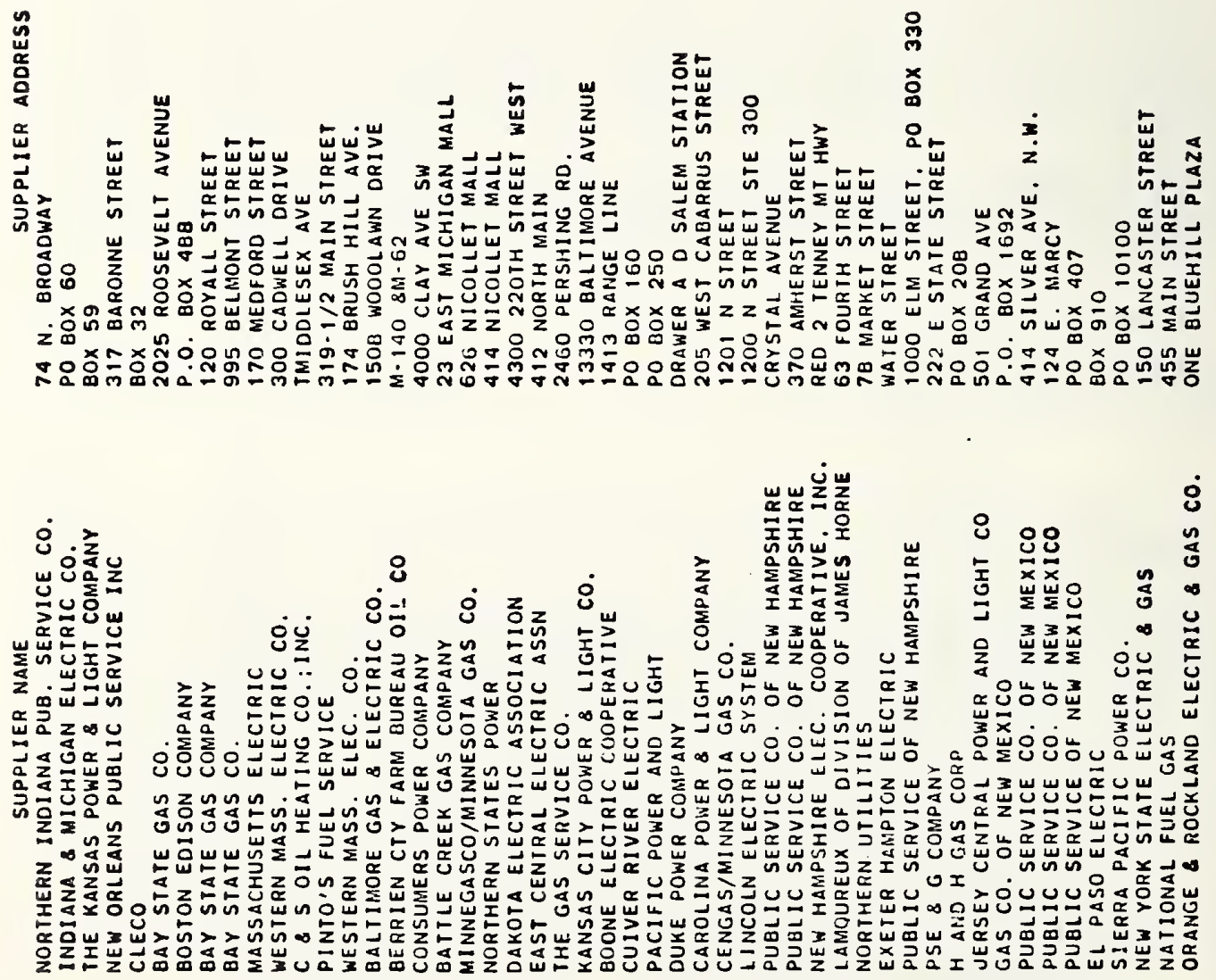

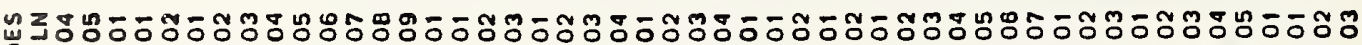

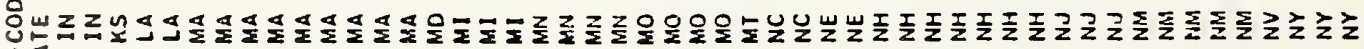
专的 


\section{DATA FILE ELEMENTS}

This section provides a detailed description of the data in the files which comprised the Solar Data Base. These data are available on tape from the National Technical Information Service (see page 59).

\section{GRANT FILE ELEMENTS}

These data were requested by HUD from organizations or individuals applying for grants for buflding homes with solar energy systems. Subsequently, data concerning applicants who received grants were stored in a file on the NBS computer. There were 668 grants awarded during the Residential Demonstration Program (1975-1980).

\section{Description of Data}

Grant ID*

Grantee Information:

Name, type and address of grantee contact person(s)

Total solar energy system cost (\$)

Portion of solar system cost requested by grantee (\$)

Project Information:

Project location address

Model Information:

Housing type

Number of dwelling units

Number of buildings

Number of solar systems

Total conditioned area per bullding

State economic area code

Solar Energy System Information:

System type (heating, cooling or water)

System kind (active, passive or hybrid)

Transfer media (air or liquid)

Solar collector - manufacturer code, type, aperture area in square feet

Auxiliary fuel type

Total cost for each solar system (\$)

Storage medium

Cost to government for ${ }^{\text {each }}$ solar system ( $\$$ )

Total load in Btu $\times 10^{6}$

Solar energy used in Btu $\times 10^{6}$

*The Grant File uses a different numbering scheme from the other files in order to comply with Federal privacy requirements. 
These data were received via three separate input forms. Grantee Reports 1, 3, and 4 were completed by grantees during different phases of the solar project. Grantee Report 1 was filed after the grant was awarded. Grantee Report 3 was filed after the construction was completed. Grantee Report 4 was filed after the building(s) or unit(s) was sold or rented.

\section{Description of Data}

\section{Grantee Report \#1}

Project ID

Project Information:

Address

Grant award date

Report \#1 date

Instrumented or non-instrumented data (yes or no)

New or retrofit

Construction Financing:

Financing arrangements (yes, no, pending)

Experience/problems

\section{Grantee Report $\$$ 3}

Dates:

Date Grantee Report 非 2 submitted (see Technical

Description file)

Date Grantee Report \#3 submitted

Final design completion date

Begin solar installation date

Solar test completion date

Construction completion date

Building Permit Data:

Approval date

Approving authority

Address

Experience/problems

Occupancy Permit Data:

Approval date

Approving authority

Address

Experience/problems

Building Codes:

National code models, if any

Local codes

Experience/problems

Construction Financing Data:

Confirmed approval date

Mortgage type 
GRANTEE FILE ELEMENTS (CONTINUED)

Period (months)

Interest rate

Amount ( $\$$ )

Financing organization

Address

Rental/Sales Agreement:

Sales/rental terms

HUD access terms

Construction Problems:

Equipment delivery problems

Equipment breakdown problems

Labor problems

Building interface problems

Other construction problems

Solar Oriented Events:

Warranty on file

Owner's manual on file

Auxiliary energy type

Grantee Report \#4

Sale Price and Mortgage Data:

Final sale price (\$)

Final rental rate $(\$)$

Mortgage amount ( $\$$ )

Period (months)

Interest rate

Mortgage approval date

Mortgage type (FHA, VA, private, etc.)

Points/fees

Mortgagor

Address

Unit status (model, sold, rental)

Report date

Initial sales price (\$)

Initial rental rate (\$)

Instrumentation (unit)

Mortgage arranged by (purchaser, builder, grantee)

Problems obtaining mortgage

Marketing Data:

Marketing period (weeks)

Date house first offered

Sales contract date

Occupancy date

Number of visitors

Number of prospective buyers

Marketing problems

General reaction by public 
Two sets of technical data were collected. The first was collected on about 25 percent of all non-instrumented systems and all of the instrumented systems. It contained a brief description of the solar energy system which was to be installed and information concerning predicted system performance. These data were basically extracted from Grantee Report $\# 2$, a report submitted by the grantee when the design of his system was completed and approved by HUD.

\section{Description of Data}

Project ID

Collector/Absorber Information:

Azimuth angle

Tilt angle

Net area - collector

Cover plate material information

Cover plate thickness

Number of cover plates

Absorber coating

Absorber substrate material

Fluid passage material

Material back insulation

Overall R-value of back insulation

Panel intercept (collector performance curve)

Panel slope (collector performance curve)

Incidence angle modifier

Freeze protection

Storage and Transport Information:

Main tank storage volume (heat)

Main tank storage volume (DHW)

Main tank storage medium (heat)

Main tank storage medium (DHW)

Pre-heat tank storage volume (heat)

Pre-heat tank storage volume (DHW)

Auxiliary tank storage volume (heat)

Auxiliary tank storage volume (DHW)

Storage temperature in main tank - upper

Storage temperature in main tank - lower

Thermal capacitance

Storage capacity

Combined heat exchanger efficiency: Collector-to-storage

Combined heat exchanger efficiency: Storage-to-load

Transport medium

Water (percent by volume)

Density

Specific heat

Flow rate 
TECHNICAL DESCRIPTION FILE ELEMENTS (CONTINUED).

Air flow correction factor

Liquid load correction factor

Backup, Heat Pump, and DHW Information:

Backup energy type - heat

Backup energy type - cooling

Backup energy type - DHW

Backup capacity - heat

Backup efficiency

Heat pump type

Heat pump - nominal capacity

Backup capacity - DHW

DHW usage

DHW set temperature

Monthly DHW inlet temperature

Miscellaneous Information:

FR-prime-tau-alpha (system performance curve)

FR-prime-UL (system performance curve)

Ventilation

Total heat loss factor - UA

CD (correction factor to be used with the degree day calculation method)

Internal heat gain

City code (reference number for local weather and insolation data)

System type

Solar Energy System Performance Information:

Available insolation - monthly

Solar contribution - monthly

Auxiliary contribution - monthly

Cooling load - monthly

Heating load - monthly

DHW load - monthly

Total load - monthly

Solar fraction - monthly

Degree days - monthly

Solar contribution - yearly

Auxiliary contribution - yearly

Cooling load - yearly

Heating load - yearly

DHW load - yearly

Total load - yearly

Solar fraction - yearly

Degree days - yearly

The second set of technical data was collected on the components of the solar energy system to be instrumented. Predicted performance data, 
TECHNICAL DESCRIPTION FILE ELEMENTS (CONTINUED)

schematics of the system and a site drawing were also included. These data were recorded on microfilm or microfiche.

Description of Data

System Schematics and Site Drawings

Project ID

Bullding and Site Description:

Project location

Building designer

Mechanical designer

Solar system designer

General contractor

Mechanical contractor

Solar contractor

Other participants (if any)

Design integration monitor

Type of solar system integration

Regulatory codes

Name and year of state or local code/regulation

Model codes which are the basis for regulations

General Characteristics:

Building type

Planned type of ownership

Planned type of occupancy

Site:

Development type

Latitude

Longitude

Altitude

Average summer temperature

Average winter temperature

Heating Design Temperatures:

Out door

Indoor

Cooling Design Temperatures:

Outdoor

Indoor

Building:

Front of building faces (direction)

Average number of stories above ground

Average number of stories below ground 
Total helght above grade

Total conditioned floor area

Roof type

Design Heat Loss/Load and Related Building Data:

Design heat loss/load to design conditions

Heat loss/load calculation method

Attic

Design shaded glass areas

Bullding ventilation rates

Internal heat gain assumptions

Site:

Heating degree days per month

Insolation per month

Heating degree days per year

Solar System Description:

System ID:

Firm

Model name/number

Type of system:

Air, active

Air, passive

Liquid, active

Liquid, passive

System and component summary:

Number of collector types

Number of circulation loops

Number of thermal storage units

Number of operational modes

Number of pumps

Number of valves

Number of blowers

Number of dampers

Number of sensors

Number of flow regulators

Number of pressure regulators

Number of subsystem fail-safe controls

Solar System Cost and Lifetime Estimates:

System design life

Design life collector 非

Design life collector \#2

Equipment Costs:

Collectors ( $\$$ )

Storage units ( $\$$ )

Distributon and controls ( $($ ) 
TECHNICAL DESCRIPTION FILE ELEMENTS (CONTINUED)

Other Costs:

Installation costs (\$)

Other ( $\$$ )

Collector:

Identification (manufacturer, address)

Model name/number

Type

Location, orientation, tilt

Array and collector characteristics

Collector shading

Cover Plates:

Number of cover plates

Location

Manufacturer

Product name/number

Material

Physical dimensions

Optical properties

Edge or surface treatment

Absorber:

Identification

Material

Number of absorbers per collector

Coating

Heat transfer fluid passages

Insulation:

Layer one - sides

Layer two - sides

Layer one - back

Layer two - back

Gaskets and Sealants:

Location

Material (sealants)

Material (gaskets)

Frame:

Identification

Material

Protective coating

Standoffs

Number of structural attachment points

Bullt-in collector 


\section{Reflector:}

Identification

Number of reflectors

Substrate material

Reflective coating

Protective coating

Physical dimensions

Other Information:

Desiccant

Freeze protection

Overheating protection

Passive collector heat transfer control

\section{Collector Performance}

Thermal Storage Unit

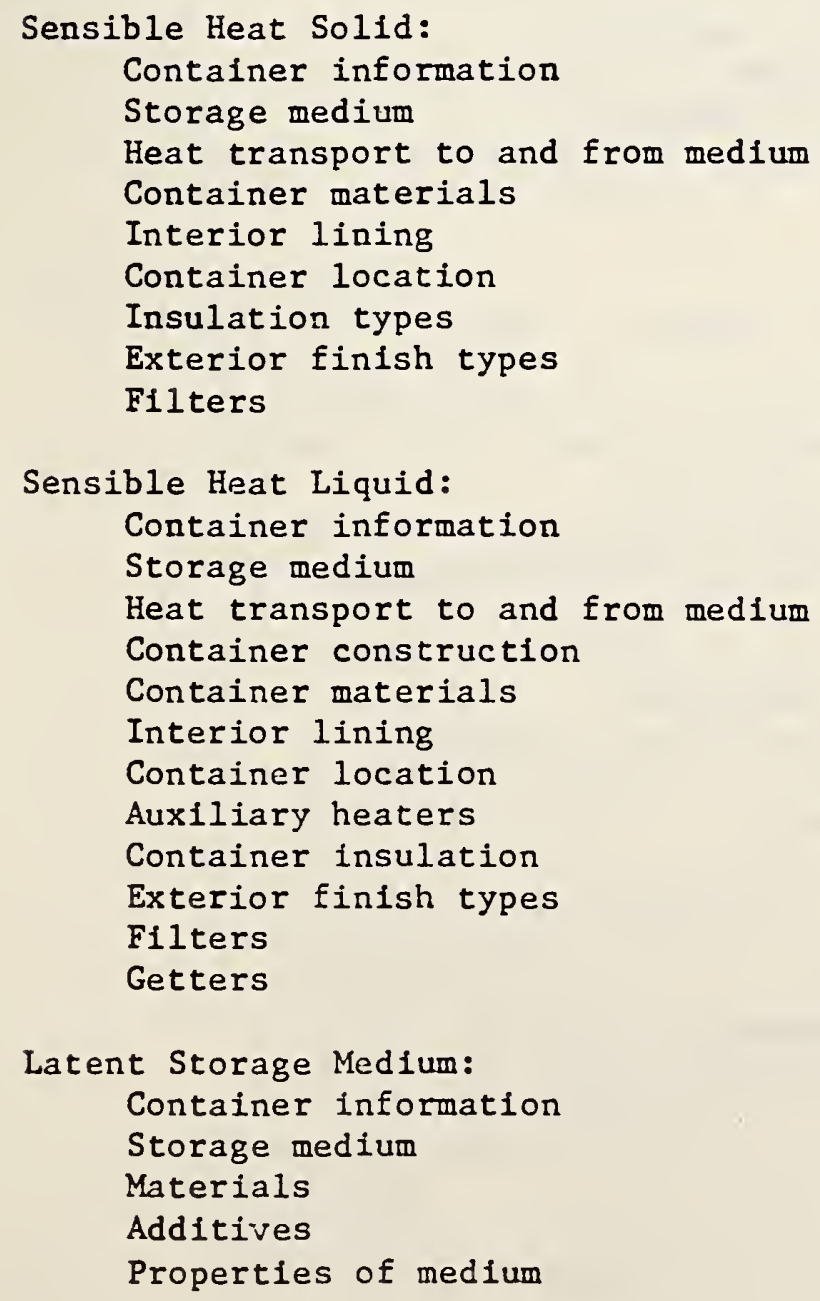




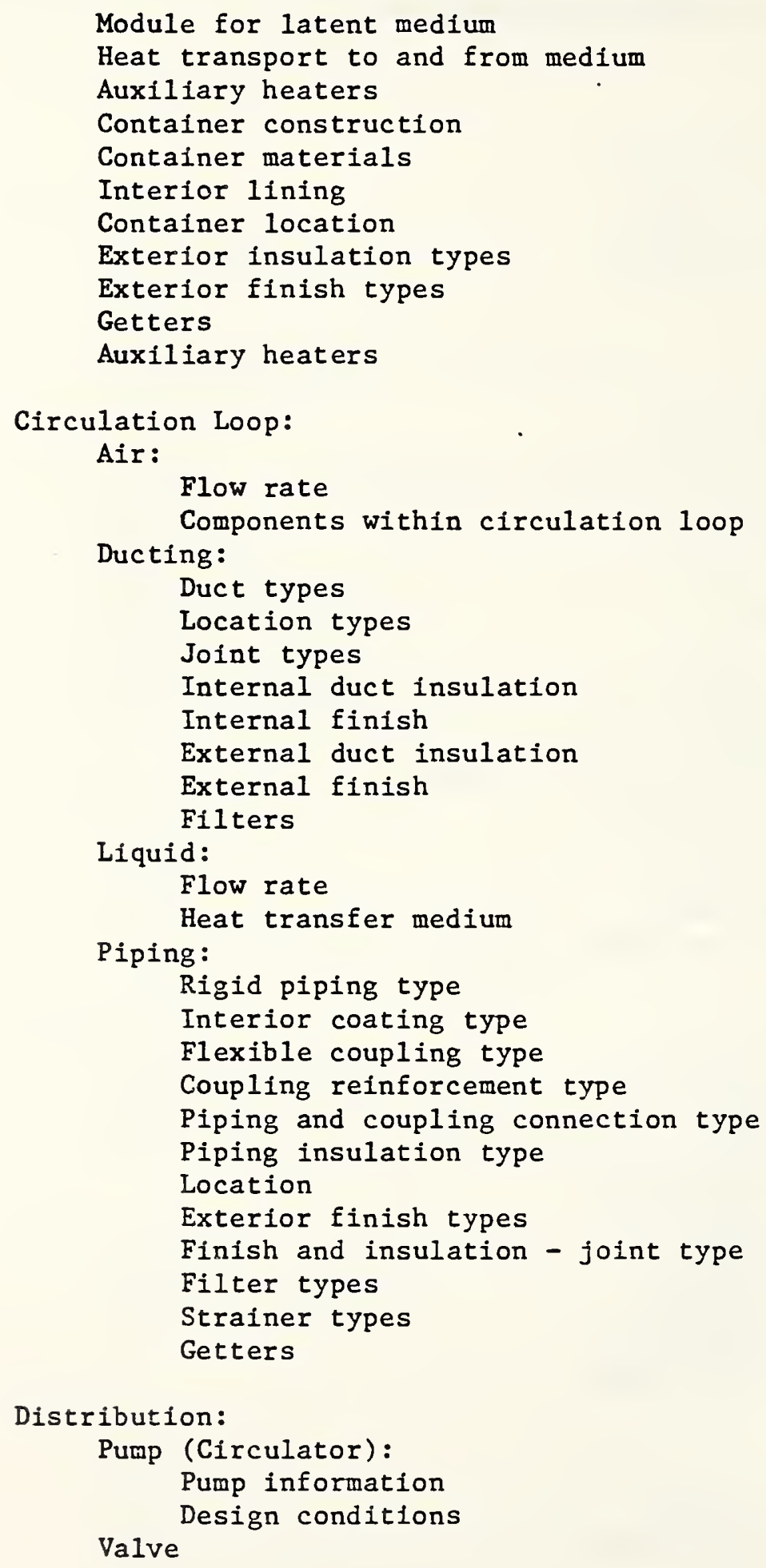




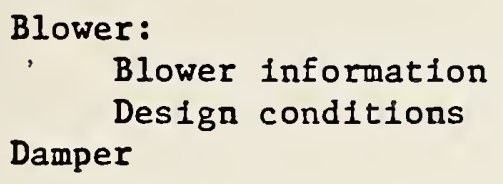

Heat Exchanger:

Air to air

Air to liquid:

Material types

Heating

Cooling

Liquid to liquid:

Material types

Heating

Cooling

Air to refrigerant

Liquid to refrigerant

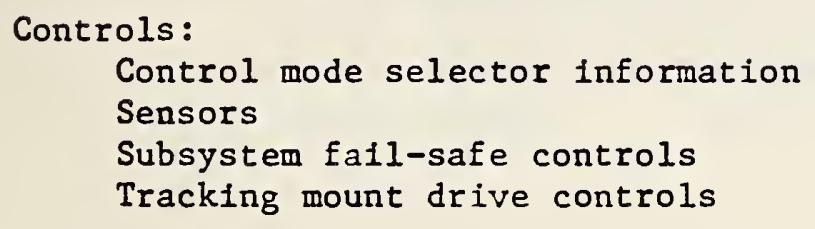

Control mode selector information

Sensors

Subsystem fall-safe controls

Tracking mount drive controls

Auxiliary Energy:

Domestic water heater:

Energy source

Burner ignition method

Automatic flue vent

Furnace:

Energy source

Burner ignition method

Automatic flue vent

Electric resistance heaters

Boiler:

Energy source

Burner ignition method

Automatic flue vent

Air conditioning:

Air conditioning information

Refrigeration machine:

Description

Operating characteristics

Burner ignition method

Automatic flue vent

Heat rejection device

Dehumidifiers:

Description

Operating conditions 
TECHNICAL DESCRIPTION FILE ELEMENTS (CONTINUED)

\author{
Humidifiers: \\ Description \\ Operating conditions \\ Supplemental heater \\ Heat pumps (reverse cycle air conditioner): \\ Type \\ Heating mode \\ Cooling mode \\ Heat pumps (reverse cycle refrigeration machine): \\ Description \\ Heat pump heat exchanger: \\ Liquid to refrigerant \\ Air to refrigerant. \\ Predicted System Performance: \\ Space temperature (heating) \\ Space temperature (cooling) \\ Domestic hot water temperature \\ Total demand load (MMBtu) \\ Energy supplied by solar system (MMBu) \\ Energy supplied by auxiliary systems (MMBU) \\ Solar system operating energy ( $\mathrm{kWh}$ ) \\ Simulation time period
}




\section{TECHNICAL CONCERNS FILE ELEMENTS}

Data for this file were generated when a technical representative made contact with a solar project where.technical concerns were being experienced. These technical concerns ranged from minor concerns (such as delivery delays due to weather) to significant concerns (such as outgassing due to faulty material selection). Selected reports documenting technical concerns during design, construction, or operational phase of the project were transcribed and put onto the computer.

\section{Description of Data}

Project ID

System Number

Date of Contact

Hardware Element With a Technical Concern

Action Taken (i.e., repair, replace, etc.)

Event(s) Which Caused Technical Concern (i.e., breakage, delivery delay, etc.)

Action Taken by HUD, Grantee, etc.

Direct Cost of Action (\$)

Performance Area (thermal, structural, mechanical, etc.)

Project Status (completed or action pending)

Phase (design, construction, operation)

Cycle Number

Type of Failure Which Caused the Technical Concern (catastrophic to questionable)

Frequency of Technical Concern 


\title{
MARKETING SURVEY FILE ELEMENTS
}

This file contains survey researci data from builders, lenders, zoning officials, solar homebuyers, $\equiv d$ other market participants. Data are non-tecinical and designed to proilde marketplace and attitudinal information as well as perceptions o: constraints on the entry of solar energy to the residential housing maziet.

The data were obtained using one or a ccabination of 26 sets of interview questionnaires. Most collscted ic Eormation was used in several studies and analyses, includi=s stucies of building code regulations, economic performance modeling, financial feasibility, consumer attitudes, legal issues, and land use. Abot: 25 percent of all grants were included in this sample. The seae set of grants had technical description and utility consumption cata coilected.

\section{Description of Data}

\author{
Single Family Builder/Developer \\ Comparative Single Family Builder/Derelcser \\ Multi-Family Builder/Developer \\ Comparative Multi-Family Builder/Develop?r \\ Purchaser \\ Comparative Purchaser \\ Prospective Purchaser \\ Renter \\ Comparative Renter \\ Building Management \\ Participating Construction Lender \\ Participating Permanent Lender \\ Non-Participating Lender \\ Insurance Company \\ Utility Company (Auxiliary) \\ Utility Company (Alternative) \\ Local Planning and Zoning Official \\ Local Building Code Official \\ Local Tax Assessor \\ Purchaser Follow-Up \\ Comparative Purchaser Follow-Up \\ Renter Follow-Up \\ Comparative Renter Follow-Up \\ Participating Builder Follow-Up \\ Comparative Builder Follow-Up \\ Site/House Description
}




\section{UTILITY CONSUMPTION FILE ELEMENTS}

The utility consumption reports contain data solicited from utility companies which supply service to grant units. Information regarding auxiliary (non-solar) fuel consumed by housing units equipped with solar devices was collected along with comparative fuel consumption data on non-solar equipped units of similar size and design. About 25 percent of all grants contained systems for which utility consumption data were collected. This sample corresponded to the Marketing Survey File sample.

\section{Description of Data}

Project ID

Supplier:

Auxiliary energy type

Meter number

Supplier code

\section{Billing Information: \\ Start of billing period \\ End of billing period \\ Present meter reading \\ Previous meter reading \\ Energy consumed \\ Billing frequency}

Cost Information:

Rate code

Energy cost ( $\$$ )

Surcharge, if any

Tax

Total cost this period (\$) 


\section{INTERACTIVE ACCESS TO SOLAR DATA}

Some users of the data which was collected in the Residential Solar Heating and Cooling Demonstration Program (those specifically authorized by HUD to do so) were able to directly access interactive files of the solar data base (see figure 2, page 51) through remotely situated computer terminals which were tied to the main computer at the National Bureau of Standards by telephone lines. The Solar Data Center (SDC) had provided access to the files through MIRADS (Marshall Information Retrieval and Display System).

MIRADS is an online storage and retrieval system generally used for retrieval of non-technical data, such as Grant File data. Under MIRADS, in response to the issuance of four basic commands: QUERY, SORT, COMPUTE, and PRINT; the system searches the data base (based on the selection criteria in the query command), sorts and retrieves selected data as specified, performs any computations requested, and prints the results.

The SDC had written the MIRADS User's Guide [6] for users of the interactive solar files. It contains the basic MIRADS language rules, examples of use, and a step-by-step walk-through of a typical interactive session. 


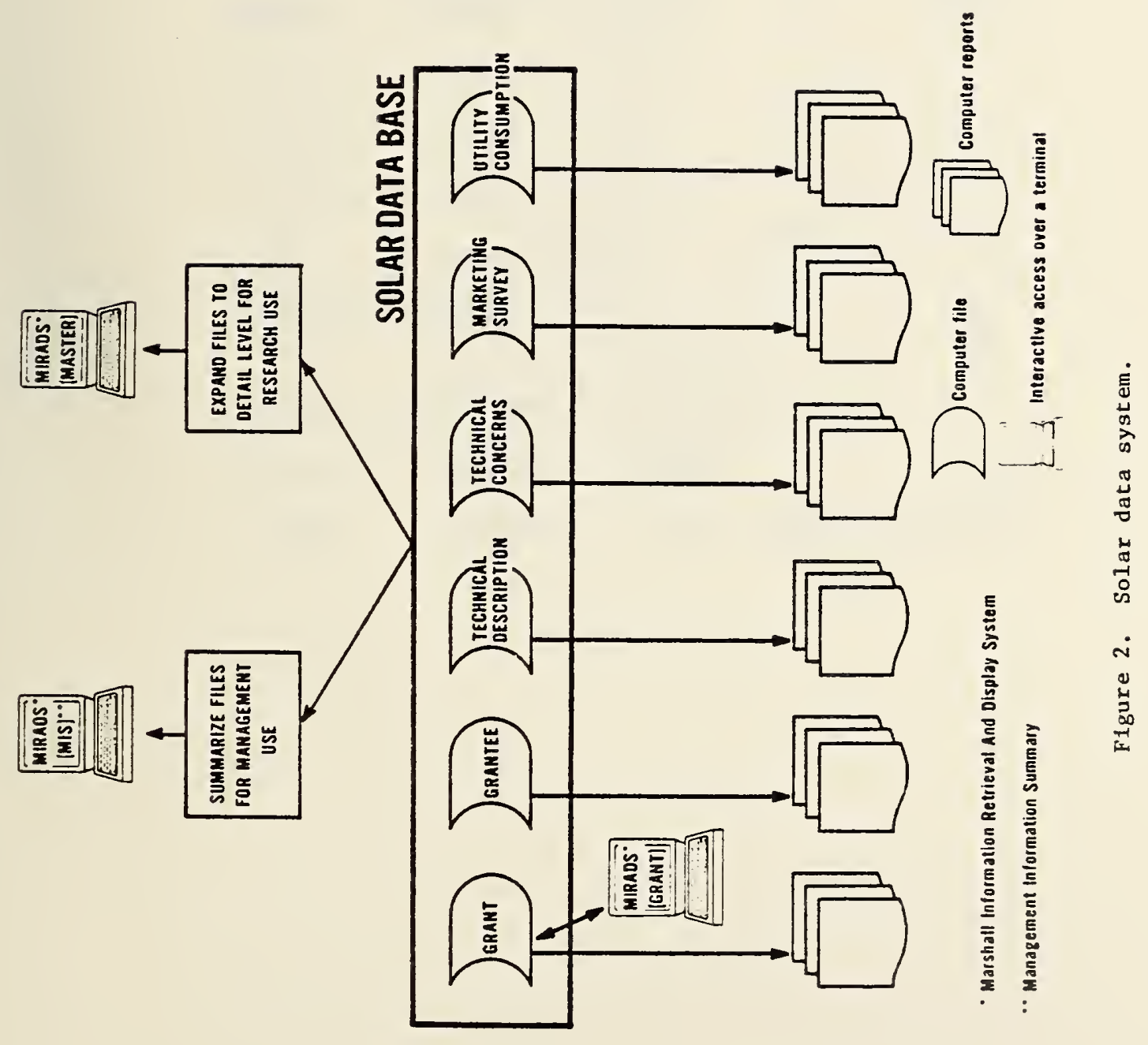




\section{AMOUNT OF DATA COLLECTED}

The tables in this section show the amount of data collected during the Residential Solar Demonstration Program from 1975-1981.

Table 7. Summary of Grant File Data

\begin{tabular}{lrrrr} 
Cycle & Grants & & $\begin{array}{c}\text { Dwel1ing } \\
\text { Units }\end{array}$ & Systems \\
\cline { 2 - 2 } 1 & 50 & 135 & 113 \\
2 & 70 & 1290 & 253 \\
3 & 145 & 3093 & 415 \\
4 & 36 & 1709 & 58 \\
$4 A$ & 75 & 3617 & 236 \\
5 & 130 & 175 & 101 \\
P1 & 162 & 79 & 79 \\
TOTAL ALL CYCLES: & 668 & 10,098 & 1255
\end{tabular}


Table 8. Summary of Grantee File Data

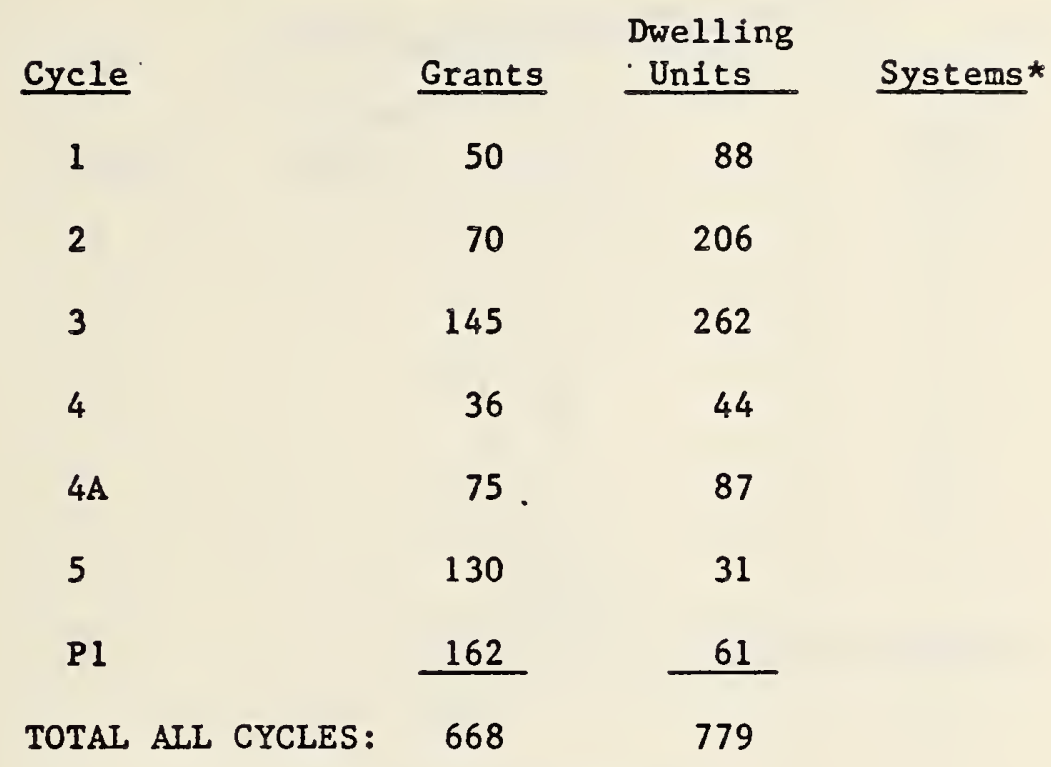

*Data not collected at this level. 


\begin{tabular}{|c|c|c|c|c|}
\hline \multicolumn{5}{|c|}{ Non-instrumented Systems } \\
\hline Cycle & & Grants & $\begin{array}{c}\text { Dwelling * } \\
\text { Units } \\
\end{array}$ & Systems \\
\hline 1 & & 26 & & 43 \\
\hline 2 & & 44 & & 168 \\
\hline 3 & & 60 & & 154 \\
\hline 4 & & 9 & & 30 \\
\hline $4 \mathrm{~A}$ & & $12^{\circ}$ & & 33 \\
\hline TOTAL ALL & CYCLES : & 151 & & 428 \\
\hline
\end{tabular}

\section{Instrumented Systems}

\begin{tabular}{|c|c|c|c|}
\hline Cycle & Grants & $\begin{array}{l}\text { Dwelling* } \\
\text { Units }\end{array}$ & Systems \\
\hline 1 & 6 & & 10 \\
\hline 2 & 26 & & 31 \\
\hline 3 & 19 & & 26 \\
\hline 4. & 2 & & 3 \\
\hline $4 \mathrm{~A}$ & 4 & & 5 \\
\hline PI & 4 & & 5 \\
\hline TOTAL ALL CYCLES: & 61 & & 80 \\
\hline
\end{tabular}


Table 10. Summary of Technical Concerns File Data

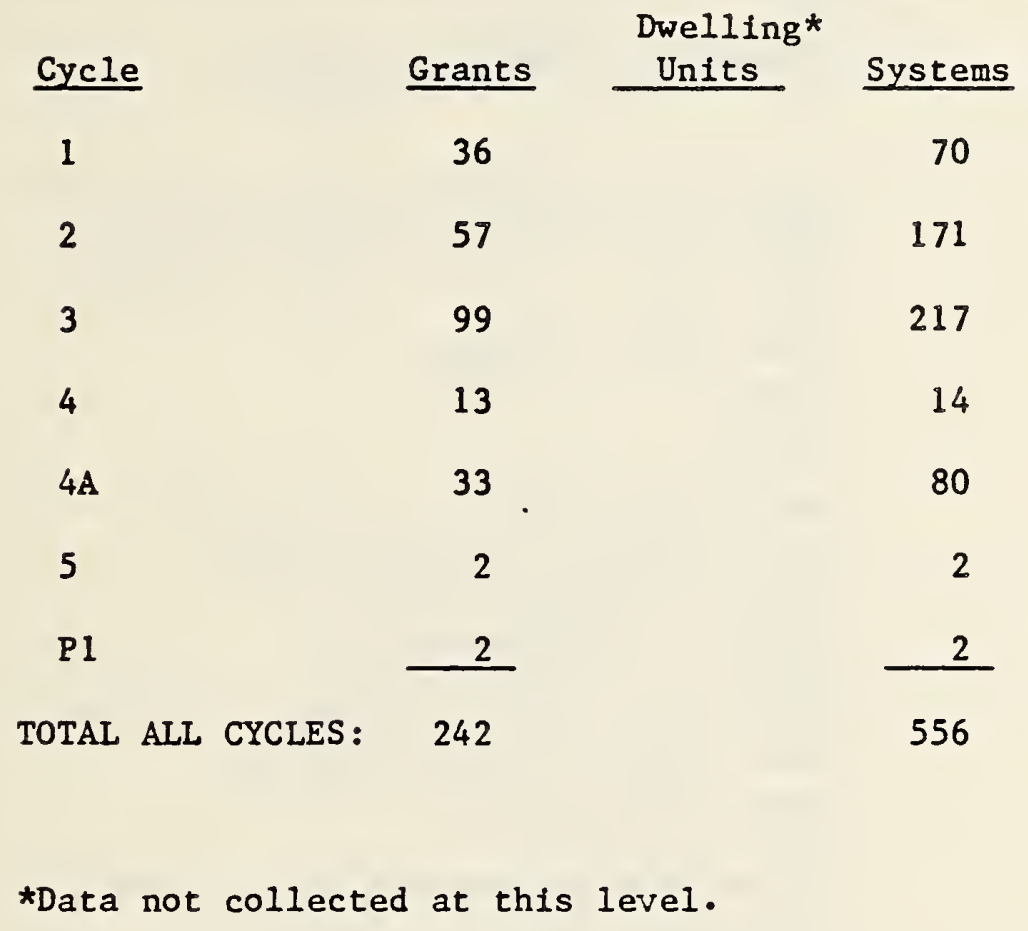


Table 11. Summary of Marketing Survey File Data

$\underline{\text { Reference }}$

Questionnaire Administered To:

Sample Size

RA

Single-Family (SF) Builder or Developer

138

$R B$

RC*

Comparative SF Builder or Developer

260

$\mathrm{RE}$

Multi-family (MF) Builder or Developer

Purchaser

276

Comparative Purchaser

252

RG

Prospective Purchaser

RH*

Renter

$\mathrm{RJ} *$

Comparative Renter

$\mathrm{RL}$

Participating Construction Lender

105

RM

Participating Permanent Lender

$\mathrm{RN}$

Non-participating Lender

129

Insurance Company/Agency

112

RP

Auxiliary Utility Company

92

Alternative Utility Company

43

$R Q$

$R R$

Local Planning/Zoning Official

105

RS

Local Building Code official

104

Local Tax Assessor

68

Follow-up Builder

121

$\mathrm{RU}$

$\mathrm{RV*}$

Follow-up Comparative Builder

137

Follow-up Comparative Purchaser

28

Follow-up Purchaser

173

RW

RW2

Second Follow-up Purchaser

117

Third Follow-up Purchaser

51

House/Site Description

531

* This data is available from RERC only in hard-copy files.

** The house/site description sample is approximately $80 \%$ of all possible single family, for-sale grants. 
Table 12. Summary of Utility File Data

\begin{tabular}{ccccc} 
Cycle & Grants & $\begin{array}{c}\text { Dwelling } \\
\text { Units }\end{array}$ & $\begin{array}{c}\text { Comparative } \\
\text { Dwelling } \\
\text { Units }\end{array}$ & Systems* \\
\cline { 2 - 3 } 2 & 26 & 65 & 26 & 30 \\
3 & 32 & 105 & 36 \\
4 & 41 & 64 & 4 \\
4 A & 6 & 9 & 0 \\
5 & 4 & 9 & 0 \\
P1 & 2 & 29 & -96 \\
TOTAL ALL CYCLES : & 139 & 283 & 29
\end{tabular}

*Data not collected at this level. 


\section{ACKNOWLEDGMENTS}

The authors wish to thank William Freeborne of the Department of Housing and Urban Development for his review of this document and his helpful suggestions and comments.

The authors would also like to acknowledge the contribution of Joan E. Krzewick of the National Engineering Laboratory, NBS, in co-authoring the earlier, 1979 version of this publication. 


\section{REFERENCES}

[1] Solar Data Center status reports, SDC Report No. 4; 1978 January and October.

(2) Christopher, Patricia M.; Krzewick, Joan E. Residential solar data center: data resources and reports. Nat. Bur. Stand. (U.S.) NBSIR 79-1762; NTIS/PB297582; 1979 June. 61 p.

[3] Aronoff, M. J.; Deutsch, D. R. Project plan for development of data center. ICST Planning Report No. 1; 1977 January.

Ruthberg, Z. G. Estimation of input data. ICST Planning Report No. 2; 1977 January.

Collica, J. C. NBS computer resources meeting data center requirements. ICST Planning Report No. 3; 1977 March.

Fong, E. Design of data dictionary. ICST Planning Report No. 4; 1977 March.

Leong-Hong, B.; Marron, B. User's manual for online retrieval of grant application data. ICST Planning Report No. 5; 1977 August.

[4] Christopher, Patricia M.; Charlton, Lynne Residential solar data center grant reports. Nat1. Bur. Stand. (U.S.) NBSIR 81-2376; 1981 September. 136 p.*

[5] Christopher, Patricia M. Residential solar data center: data dictionary/directory. Natl. Bur. Stand. (U.S.) NBSIR 81-2357; 1981 September. 94 p.*

[6] Christopher, Patricia M.; Vogt, Michael; Hall, Douglas Residential solar data center MIRADS user's guide. Natl. Bur. Stand. (U.S.) NBSIR 80-2144; 1980 October. 134 p.*

*Will be available from: National Technical Information Service 5285 Port Royal Road Springfield, VA 22161 

. 


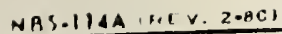

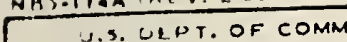

BIBLIOGRAPHIC DATA

SHEET (See instructions)

1. PUBLICATION OR REPORT NO.

NBSIR $81-2369$

2. Performing Organ. Report Nof 3. Publication Date

October 1981

4. TITLE AND SUBTITLE

Residential Solar Data Center: Data Resources and Reports

3. $A \cup T H O R(S)$

Patricia $M$. Christopher and Audrey 0 . Houser

6. 'ERIORMING ORGANIZATION (If joint or other than NBS, see instructions)

MATIONAL BUREAU OF STANDAROS

DEPARTMENT OF COMMERCE

WASHINGTOH, D.C. 20234

7. ContracuGrant No.

IAA-H-54-81 (formerly

8. Hype of Repor? \& Period Covered Final

1. 'PUUSSORING ORGANIZATION NAME AND COMPLETE ADDRESS (Street, City. State, ZIP)

Sept. 1979 to Sept. 1981

Department of Housing and Urban Development

Office of the Assistant Secretary for Policy Development and Research

Division of Energy, Building Technology and Standards

hashington, D.C. 20410

10. SUPPLEMENTARY NOTES Supersedes NBSIR 79-1762, Residential Solar Data Center: Data Resources
and Reports

Document describes a computer program; SF-185, FIPS Software Summary, is attached.

11. ABSTRACT (A 200-word or less foctuol summory of most significant information. If document includes a significant tiblion:rophy or literoture survey. mention it here)

The Residential Solar Data Center (SDC) was responsible for the establishment and operation of a computerized data base containing non-instrumented residential data collected from the DoE/HUD Solar Heating and Cooling Demonstration Program. This document includes a sumnary of the history and background of the SDC and its role in the demonstration program, a list of the final computer reports which are avallable, sample pages of representative reports, and a description of the data files which comprised the solar data base.

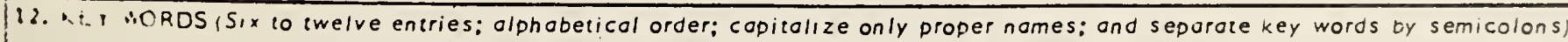

Automatic data processing; data base; residential buildings; solar data base; 11. $\therefore$ in beatirg and coling; solar energy systems

Unlimiled

For Otricial Distribution. Do Not Release to NTIS

: ier From Suderintendent of Documents, U.S. Government Printing Office. Washington, D.C.

X C.uer From National Technical Information Service (NTIS). Springfield. VA. 22161

14. NO. OF PRINTED PAGES

65

15. Price 


